\title{
Using atomic layer deposition to hinder solvent decomposition in lithium ion batteries: first principles modeling and experimental studies
}

\author{
Kevin Leung, ${ }^{1 *}$ Yue Qi, ${ }^{2}$ Kevin R. Zavadil, ${ }^{1}$ Yoon Seok Jung, ${ }^{3,7}$ Anne C. \\ Dillon $^{3}$, Andrew S. Cavanagh, ${ }^{4}$ Se-Hee Lee, ${ }^{5}$, and Steven M. George ${ }^{6}$ \\ ${ }^{1}$ Sandia National Laboratories, \\ $M S 1415 \& 30888$, \\ Albuquerque, NM 87185 \\ *kleung@sandia.gov \\ ${ }^{2}$ General Motors RES D Center. Warren, \\ MI 48090, U.S.A. \\ ${ }^{3}$ National Renewable Energy Laboratory, \\ Golden, CO 80401 \\ ${ }^{4}$ Department of Physics, \\ University of Colarado, \\ Boulder, CO 80309 \\ ${ }^{5}$ Department of Mechanical Engineering, \\ University of Colarado, \\ Boulder, CO 80309 \\ ${ }^{6}$ Department of Chemistry and Biochemistry, \\ University of Colarado, Boulder, \\ ${ }^{7}$ Interdisciplinary School of Green Energy, \\ Ulsan National Institute of Science and Technology (UNIST), \\ Ulsan 689-798 South Korea
}

(Dated: June 5, 2018) 


\begin{abstract}
Passivating lithium ion battery electrode surfaces to prevent electrolyte decomposition is critical for battery operations. Recent work on conformal atomic layer deposition (ALD) coating of anodes and cathodes has shown significant technological promise. ALD further provides wellcharacterized model platforms for understanding electrolyte decomposition initiated by electron tunneling through a passivating layer. First principles calculations reveal two regimes of electron transfer to adsorbed ethylene carbonate molecules (EC, a main component of commercial electrolyte) depending on whether the electrode is alumina-coated. On bare Li metal electrode surfaces, EC accepts electrons and decomposes within picoseconds. In contrast, constrained density functional theory calculations in an ultra-high vacuum setting show that, with the oxide coating, $e^{-}$ tunneling to the adsorbed EC falls within the non-adiabatic regime. Here the molecular reorganization energy, computed in the harmonic approximation, plays a key role in slowing down electron transfer. Ab initio molecular dynamics simulations conducted at liquid EC-electrode interfaces are consistent with the view that reactions and electron transfer occur right at the interface. Microgravimetric measurements demonstrate that the ALD coating decreases electrolyte decomposition and corroborate the theoretical predictions.
\end{abstract}




\section{INTRODUCTION}

Improving the fundamental scientific understanding of lithium ion batteries ${ }^{\underline{1}-3}$ is critical for electric vehicles and other energy storage technologies. A key feature that enables the use of negative electrodes (graphite, Li metal, $\mathrm{Si}, \mathrm{Sn}$ ) operating below the reduction voltage of current commercial electrolytes is the formation of an electronically passivating but $\mathrm{Li}^{+}$-conducting solid electrolyte interphase (SEI) film on electrode surfaces $\underline{\underline{1}}^{-\underline{\underline{5}}}$ Battery performance, irreversible capacity "loss," power fade, durability, exfoliation of graphite, and safety are highly dependent on the quality of the SEI. Therefore understanding the nature, formation composition, structure, and property of SEI is of great interest for Li-ion batteries. In this work, we apply computational and experimental techniques to analyze the success of the conformal atomic layer deposition (ALD) strategy for creating a passivating layer ("artificial SEI") on electrodes, $\underline{\underline{6}-\underline{\underline{10}}}$ focusing on graphitic carbon anodes. $\underline{\underline{11}}$

It is generally accepted that, upon the first charge of uncoated graphitic anodes, the negative potential applied to induce $\mathrm{Li}^{+}$intercalation into graphite decomposes ethylene carbonate (EC) molecules in the solvent, yielding a self-limiting, 3-10 nm thick, passivating SEI layer containing $\mathrm{Li}_{2} \mathrm{CO}_{3}$, lithium ethylene dicarbonate $\left(\left(\mathrm{CH}_{2} \mathrm{CO}_{3} \mathrm{Li}\right)_{2}\right)_{,}, \underline{2,4}, \underline{5}$ and salt decomposition products. Early modeling work on organic solvent breakdown has focused on reactions inside bulk liquid regions, with an excess electron already injected. $\underline{12} \underline{15}$ While providing extremely useful predictions pertinent to that regime, such models necessarily ignore the possibility of surface-assisted reactions and effects arising from electron transfer from electrodes. A more rigorous if costly technique, ab initio molecular dynamics (AIMD), has recently been applied to simulate chemical reactions at several explicit solidliquid interfaces. $\underline{16}-20$ One of the authors' previous AIMD works follows chemical reactions in real time at the pristine graphitic anodes/liquid EC interface. ${ }^{21}, 22$ It is found that, at the initial stage of SEI formation, fast $e^{-}$transfer and kinetically-controlled EC electrochemical reactions occur to form either $\mathrm{CO}$ or $\mathrm{C}_{2} \mathrm{H}_{4}$ gas, $\stackrel{23-26}{2}$ mostly right at the oxidized edges of graphite sheets. $\stackrel{27}{2}, 28$

As electrolyte decomposition proceeds, $e^{-}$transfer becomes impeded by the intervening and partially-formed SEI layer between the solvent and electrode, and the decomposed solvent fragments can no longer anchor directly to the pristine electrode surface. This important next stage should figure equally prominently in the overall solvent breakdown 
mechanism and the structure of naturally-formed SEI. The electron tunneling blockage by SEI layers is a kinetic (not thermodynamic) phenomenon, akin to stoppage of electron leakage through gate-oxide dielectric in semiconductor devices. ${ }^{29}$ Theoretical study there is hindered by the substantial thickness, possibly porous/gel-like nature, and heterogeneous composition of natural SEI. ${ }^{-3}, 30$

Recently, it has been demonstrated that a sub-nanometer thick alumina layer created by the conformal ALD technique on graphite drastically diminishes solvent decomposition but permits lithium ion transport $\underline{11}$ This promising ALD strategy even enables the cycling of low-melting-point propylene carbonate (PC), which otherwise exfoliates and destroys uncoated graphitic anodes. The mechanism of this ALD electrode passivation has not been completely understood. While expected to block or slow down electron transfer from the electrode to the solvent, it also appears to enhance the mechanical properties of the electrodes, $\frac{6}{6}$ and likely hinders solvent intercalation between graphite sheets, thus preventing exfoliation. Apart from the technological implications, the unprecedented control over coating thickness and chemistry means that the ALD strategy also provides robust platforms for basic science studies of interfacial solvent decomposition reaction mechanisms, and for electron tunneling through the insulating layer which is a pre-requisite for electrolyte breakdown.

In particular, the extreme thinness of ALD coatings lends itself to the present, predominantly first principles computational study of electrode/solvent interfaces comprising up to 850 atoms. Using crystalline, hydroxylated $\mathrm{LiAlO}_{2}$ layers as models of ALD coatings, we apply DFT and related theoretical techniques to show that ALD oxide films yield varying energetic, kinetic, and electron-tunneling impedence towards EC breakdown depending on the surface Li content and oxide thickness. We also provide evidence that $e^{-}$transfer occurs to EC molecules immediately next to electrode surfaces. Because these molecules are deep within the electric double layer (EDL), screening of electric fields by the EDL is less effective. In this sense, EC decomposition at battery anodes can differ fundamentally from classic electrochemical redox paradigms, where well-solvated transition metal complexes are separated by several Angstroms from the electrodes and "outer-shell" $e^{-}$tunneling dominates. 31 The solvent decomposition processes on ALD coatings provide insights that may be extrapolated to other passivation strategies, including natural SEI formed from electrolyte breakdown.

Two limiting regimes of electron transfer, and two corresponding computational methods, are emphasized. Rigorously, DFT deals with the electronic ground state, with nuclear 
trajectories "adiabatic" to electronic configurations (i.e., ionic motions are slow compared with electron transfer). EC breakdown on $\mathrm{Li}$ (100) metal surface is in this adiabatic regime. DFT should be adequate for such processes, provided that the desired electronic configuration is the ground state and the self-interaction error of the approximate functional used is not critical to the properties being investigated $\underline{32}^{32}$

In the opposite, non-adiabatic regime, $\stackrel{37-\underline{41}}{\underline{4}} e^{-}$transfer or tunneling is slow on the time scale of nuclear motion, and one must keep track of two electronic surfaces. ${ }^{31}$ The oxidecoated model electrodes considered in this work pertains to this latter limit, where the electron transfer rate between two discrete orbitals is given by ${ }^{42}$

$$
k_{\mathrm{et}}=\frac{\sqrt{\pi}\left|V_{\mathrm{AB}}\right|^{2}}{\hbar \sqrt{\lambda k_{\mathrm{B}} T}} \exp \left[-\frac{\left(\Delta G_{o}+\lambda\right)^{2}}{4 \lambda k_{\mathrm{B}} T}\right] .
$$

$\lambda$ is the reorganization (free) energy, $V_{\mathrm{AB}}$ is the coupling matrix element connecting the two electronic surfaces, and $\Delta G_{o}$ is the reaction free energy. $\lambda$ indicates the energy cost associated with molecular deformation needed to take on an extra electron $\left(\mathrm{EC} \rightarrow \mathrm{EC}^{-}\right)$. $V_{\mathrm{AB}}$ is the familiar prefactor that depends on the overlap between two many-body wavefunctions associated with the two electronic surfaces (Fig. 1a). Small $V_{\mathrm{AB}}$ correlates with non-adiabatic $e^{-}$tunneling.

Neither $V_{\mathrm{AB}}$ nor $\lambda$ can be directly obtained using standard DFT methods. In this work, $\lambda$ is estimated using the constrained DFT (cDFT) approach $\underline{38,43}$ and Marcus theory harmonic construction (Fig. 1a) under both ultra-high vacuum (UHV) and liquid state ${ }^{44,45}$ configurations. cDFT is also applied to estimate $V_{\mathrm{AB}} \cdot \frac{39}{}$. While $\mathrm{cDFT}$ and related methods have been applied to molecules on metal surfaces, $\stackrel{46}{-48}$ calculating $V_{\mathrm{AB}}$ between a metallic electrode and an $e^{-}$-accepting molecule, or for that matter the total $e^{-}$tunneling rate, has relied on simplified models. $\underline{49}-\underline{55}$ When augmented using a Fermi Golden-rule expression (Fig. 1 b), we argue that the our $V_{\mathrm{AB}}$ value yields a well-defined kinetic prefactor for electron transfer from a metallic electrode. Our prefactor prediction is a preliminary estimate, and fundamental studies to extend $\mathrm{cDFT}$ to $e^{-}$transfer from metallic electrodes are needed. However, this is sufficient for our goal of order-of-magnitude estimates of $e^{-}$transfer rates. When the insulating layer (ALD oxide or natural SEI, or their combination) grows thicker, $V_{\mathrm{AB}}$ starts to decay with oxide thickness, and its magnitude is examined via extrapolation in a way analogous to the one-dimensional Wentzel-Kramers-Brillouin (WKB) formula. An alternative to this cDFT formulation may be Greens function/time-dependent DFT $\underline{56} \underline{-58}$ To our 
knowledge, TDDFT methods have not been successfully applied to predict orbital-to-orbital $V_{\mathrm{AB}}$ values that involve metallic electrodes.

With these computational techniques, we show that the sub-nanometer oxide coating,, 111 generally not considered sufficiently thick for complete electron blockage in, say, gate oxide dielectric applications, $\stackrel{29}{ }$ causes $\lambda$ (much neglected in previous battery studies) to play a significant role in ALD-assisted passivation. Electron tunneling to EC, not bond-breaking within the adsorbed molecule, is generally found to be the rate-determining step for breakdown of EC adsorbed on the ALD-coated electrode.

In terms of experiments, microgravimetric measurements that confirm the presence of solvent decomposition products on the surface are presented to corroborate aspects of our predictions.

This paper is organized as follows. Section II describes the methods used. Adiabatic electron-transfer induced EC reactions with Li metal surfaces is discussed in Sec. III. The long-range electron transfer formalism is shown to be inapplicable here. Section IV describes the non-adiabatic electron tunneling from oxide-coated electrodes to EC molecules adsorbed on their surfaces, and addresses the subsequent EC bond-breaking events. Adiabatic DFT/PBE calculations are shown to underestimate the electron tunneling barrier in this regime. Section $\nabla$ reports the experimental results, and Sec. VI briefly summarizes this work.

\section{METHODS}

\section{A. Model systems}

The casual reader is encouraged to skip forward to Sec. III for the results.

The key model systems are $\sim 7.0 \AA$ ("thin") and $\sim 10 \AA$ ("thick") layers of $\mathrm{LiAlO}_{2}$ in $\beta-\mathrm{NaAlO}_{2}$ structure with (100) surface terminations, coated on narrow strips of $\mathrm{Li}_{x} \mathrm{C}_{6}$ electrodes (Table If Fig. 2, more details of this oxide phase is provided in the supporting information (S.I.) document). Undercoordinated $\mathrm{Al}$ atoms on outer surfaces are terminated with $\mathrm{OH}$ groups, ensuring that surface states are removed. The oxide thickness is measured from $\mathrm{Al}$ to $\mathrm{Al}$ and excludes the surface hydroxyl groups or the $\mathrm{C}=\mathrm{O}$ edge atoms originally residing on $\mathrm{Li}_{x} \mathrm{C}_{6}$. Crystalline $\mathrm{LiAlO}_{2}$ is a solid-state electrolyte candidate material. $\stackrel{59}{ } \mathrm{LiAlO}_{2}$ 
is used instead of $\mathrm{Al}_{2} \mathrm{O}_{3}$ to cover the possibility that the native $\mathrm{Al}_{2} \mathrm{O}_{3}$ layers deposited during ALD may have incorporated Li ions during the first charging half cycle. For example, some $\mathrm{AlOH}$ groups may be deprotonated at low voltages, causing $\mathrm{Li}^{+}$to coordinate to the $\mathrm{AlO}^{-}$and become part of the surface. The $\mathrm{LiAlO}_{2}$ mixed oxide thus allows us to examine surface composition effects on EC breakdown. The stochiometry of the coating is such that their formal charges sum to zero. Another research group has found $\mathrm{LiAlO}_{2}$ signature on the surface of $5 \mathrm{~nm}$ thick ALD oxide films on Si anodes after power cycling using Xray photo-electron spectroscopy $\underline{60}^{6}$ Further computational evidence for Li-incorporation into $\mathrm{Al}_{2} \mathrm{O}_{3}$ films is presented in the S.I. If such $\mathrm{Li}^{+}$incorporation indeed occurs, the ALD layer will expand beyond its original $\mathrm{Al}_{2} \mathrm{O}_{3}$ thickness.

The simulation cell, which provides a modest system size for AIMD simulations, is chosen so that the oxide is fairly well-matched to the $\mathrm{Li}_{x} \mathrm{C}_{6}$ surface cell, with oxide compressive strains of $1.8 \%$ and $5.7 \%$ in the two lateral dimensions. The crystalline models are idealized; as in gate-oxide dielectric materials, insulating oxides should be amorphous to minimize cracks. The amount of $\mathrm{Li}$ present in the graphite region is determined by tuning the $\mathrm{Li}$ chemical potential to $2.1 \mathrm{eV}$. Upon geometry optimization, Li ions initially residing at the $\mathrm{C}=\mathrm{O}$ edges become strongly coordinated to the bottom surface of the oxide coating. Another model, with a single $10 \AA$ thick layer of $\mathrm{LiAlO}_{2}$ hydroxylated on both sides but no $\mathrm{Li}_{x} \mathrm{C}_{6}$ component, is used to examine post $e^{-}$-transfer $\mathrm{EC}^{-}$bond-breaking.

To emphasize the influence of surface groups, we also include a model with a $\sim 5.0 \AA$ thick layer of $\alpha-\mathrm{Al}_{2} \mathrm{O}_{3}$ coated on both sides of the $\mathrm{Li}_{x} \mathrm{C}_{6}$ strip. The oxide layers have (0001) terminations with $\mathrm{AlOH}$ surface groups (Table I). It has been predicted that $\gamma-\mathrm{Al}_{2} \mathrm{O}_{3}$ is more stable than the $\alpha$ phase for film thickness below $36 \AA .63$ However, this estimate was made without accounting for surface hydroxylation. Since our thin $\mathrm{Al}_{2} \mathrm{O}_{3}$ film contains only two Al-O layers (not counting the $\mathrm{C}=\mathrm{O}$ edge groups), the oxygen positions are arguably consistent with both $\alpha-\mathrm{Al}_{2} \mathrm{O}_{3}$ with closed packed oxygen in ABAB stacking, and cubic $\gamma$ $\mathrm{Al}_{2} \mathrm{O}_{3}$ with $\mathrm{ABCABC}$ stacking. In $\alpha-\mathrm{Al}_{2} \mathrm{O}_{3}$, all $\mathrm{Al}$ are in octahedral sites while $\mathrm{Al}$ occupy both octohedral and tetrahedral sites in $\gamma-\mathrm{Al}_{2} \mathrm{O}_{3}$. Upon applying geometry optimization to the initial " $\alpha-\mathrm{Al}_{2} \mathrm{O}_{3}$ " film, some $\mathrm{Al}$ ions are found to migrate to tetrahedral sites, especially those coordinated to graphite-edge $\mathrm{C}=\mathrm{O}$ groups. Thus our " $\alpha-\mathrm{Al}_{2} \mathrm{O}_{3}$ " film arguably exhibits both $\alpha$ and $\gamma$ character, consistent with experimental ALD coatings which are considered amorphous without long-range order. 


\begin{tabular}{|c|c|c|c|c|c|}
\hline system/coating & method & Figure & stochiometry & cell size & $\mathrm{N}_{\mathrm{EC}}$ \\
\hline thin $\mathrm{LiAlO}_{2}$ & NEB & Fig. 4c-d, 6 & $\mathrm{Al}_{48} \mathrm{Li}_{96} \mathrm{O}_{148} \mathrm{C}_{92} \mathrm{H}_{24}$ & $40.00 \times 12.47 \times 15.06$ & 1 \\
\hline thick $\mathrm{LiAlO}_{2}$ & NEB & NA & $\mathrm{Al}_{72} \mathrm{Li}_{118} \mathrm{O}_{208} \mathrm{C}_{92} \mathrm{H}_{24}$ & $46.0 \times 12.47 \times 15.06$ & 1 \\
\hline thick $\mathrm{LiAlO}_{2}$ & AIMD & Fig. 2d & $\mathrm{Al}_{72} \mathrm{Li}_{118} \mathrm{O}_{208} \mathrm{C}_{92} \mathrm{H}_{24}$ & $48.5 \times 12.47 \times 15.06$ & 36 \\
\hline thin $\mathrm{LiAlO}_{2}$ & AIMD & Fig. 2e & $\mathrm{Al}_{48} \mathrm{Li}_{96} \mathrm{O}_{148} \mathrm{C}_{92} \mathrm{H}_{24}$ & $43.00 \times 12.47 \times 15.06$ & 36 \\
\hline only $\mathrm{LiAlO}_{2}$ & NEB & NA & $\mathrm{Al}_{36} \mathrm{Li}_{36} \mathrm{O}_{84} \mathrm{H}_{24}$ & $24.00 \times 12.47 \times 15.06$ & 1 \\
\hline $\mathrm{Al}_{2} \mathrm{O}_{3}$ & AIMD & Fig. 2f & $\mathrm{Al}_{72} \mathrm{O}_{204} \mathrm{C}_{120} \mathrm{H}_{72} \mathrm{Li}_{51}$ & $33.34 \times 14.97 \times 18.82$ & 36 \\
\hline $\operatorname{Li}(100)$ & AIMD & Fig. $2 \mathrm{~b}$ & $\operatorname{Li}_{96}$ & $30.35 \times 14.63 \times 14.63$ & 32 \\
\hline $\mathrm{Li}(100)$ & NEB & Fig. 2r & $\mathrm{Li}_{96}$ & $30.35 \times 14.63 \times 14.63$ & 1 \\
\hline $\mathrm{Li}(100)$ & NEB & Fig. $3 \mathrm{~b}-\mathrm{d}$ & $\mathrm{Li}_{48}$ & $24.00 \times 9.75 \times 9.75$ & 1 \\
\hline
\end{tabular}

TABLE I: Details of systems used in AIMD and geometry optimization-plus-NEB barrier calculations. The spatial dimensions are in $\AA$. "Stochiometry" omits ethylene carbonate atoms in the liquid region.

Finally, a thin slab of lithium metal truncated along (100) surfaces is considered. Even though Li metal itself cannot currently be used as rechargeable anodes, EC breakdown products on $\mathrm{Li}$ are qualitatively similar to those on $\mathrm{LiC}_{6}$ surfaces. $\frac{1,64}{}$ Under open circuit conditions, Li metal should be at a well defined $\sim-3 \mathrm{~V}$ versus the standard hydrogen potential. ${ }^{65}$ Furthermore, EC decomposition on Li surface is free of the ambiguity associated with solvent co-intercalation into graphite. ${ }^{66}$ Thus Li metal provides an useful baseline with which to interpret predictions for the oxide-coated surfaces.

\section{B. Adiabatic regime: DFT, AIMD simulations}

All calculations are performed using the Vienna Atomic Simulation Package version 4.6 (VASP) $\underline{67,68}$ and the PBE functional. $\underline{69}$ AIMD simulations apply $\Gamma$-point Brillouin zone sampling, a $400 \mathrm{eV}$ planewave energy cutoff, and a $10^{-5} \mathrm{eV}$ or $10^{-6} \mathrm{eV}$ convergence criterion at each Born-Oppenheimer time step. The trajectories are kept at an average temperature of $\mathrm{T}=450 \mathrm{~K}$ using Nose thermostats, except for the EC/Li metal simulation where $\mathrm{T}=350 \mathrm{~K}$ is enforced. Tritium masses are substituted for protons to enable a time step of 1 fs. Under these conditions, the trajectories exhibit drifts of less than $1 \mathrm{~K} / \mathrm{ps}$. Due to the 
approximate nature of DFT functionals and the simulation protocol (tritium masses and thermostat used), the predicted reaction time scales should be treated as relative, not absolute. AIMD simulations reported do not account for spin-polarization. Our previous work has revealed no qualitative difference between restricted singlet and spin-triplet DFT/AIMD simulations. ${ }^{21}$ Molecular configurations are pre-equilibrated using Monte Carlo simulations and simple molecular force fields, as described in an earlier work. ${ }^{21}$ Representative AIMD snapshots are depicted in Fig. 2.

The AIMD liquid/solid interfacial simulations are akin to dipping electrodes fully preintercalated with Li into the organic solvent. In principle, it may be possible to intercalate $\mathrm{Li}^{+}$in the electrolyte, remove the anodes from solution, clean off possible decomposition products in inert environments, and re-insert in solution to measure the open circuit voltage. Such experiments have not been performed but can be attempted in the future. In Sec. IVD, we further discuss the electrochemical potential of these electrode models.

$\mathrm{T}=0 \mathrm{~K}$ geometry optimizations and climbing image nudged elastic band (NEB) $)^{70}$ barrier calculations (e.g., Fig. 3) are performed with spin-polarization, a $10^{-4} \mathrm{eV}$ convergence criterion, and a linear potential correction applied in the direction perpendicular to the surface to remove dipole-image interactions. ${ }^{71} \Gamma$-point sampling is generally applied, except for calculations involving Li metal slabs where $1 \times 2 \times 2$ Brillouin zone sampling is used. Even there, $\Gamma$-point NEB calculations yield a C-O bond-breaking barrier only $0.1 \mathrm{eV}$ higher than the more dense Brillouin grid result. It is also found that the geometry and net charge of an adsorbed, intact $\mathrm{EC}^{-}$on $\mathrm{LiAlO}_{2}$ surface is unchanged whether $\Gamma$-point or $1 \times 2 \times 2$ grids are used. Comparing restricted singlet and spin-polarization results, no difference is discernable in the $\mathrm{EC}$ on $\mathrm{Li}$ metal calculations, where the bond-breaking barrier is small $(<0.1 \mathrm{eV}$, Sec. III) and adiabatic electron transfer from the electrode and the bond-breaking event occur simultaneously. These are the conditions under which spontaneously EC decompositions are observed in picosecond AIMD simulations, justifying the use of non-spin-polarized DFT there. Higher bond-breaking barriers, like those on the $10 \AA$ thick $\mathrm{LiAlO}_{2}$ surface (see the S.I.), are reduced when spin polarization is allowed. A spot check shows that spinunrestricted DFT calculations reduce the $\mathrm{C}_{\mathrm{C}}-\mathrm{O}$ cleavage barrier by $0.15 \mathrm{eV}$ on this surface. Even with this reduction, the barrier is high enough to prevent observation of EC breakdown in picosecond time scale, and therefore using non-spin-polarized DFT in AIMD simulations does not affect the conclusion that no reactions occur within the 7 ps trajectories in high 
barrier cases. Further details on NEB calculations are discussed in the S.I.

\section{Non-adiabatic regime: Constrained DFT}

A version of the constrained DFT (cDFT) method $\underline{38,39}$ is implemented into VASP. The constraining potential is chosen to be

$$
\begin{aligned}
W(\mathbf{r}) & =V_{o}\left[1-\Pi_{i} f_{i}(\mathbf{r})\right], \\
f_{i}(\mathbf{r}) & =\left[1+\tanh \left(\kappa\left(\left|\mathbf{r}-\mathbf{r}_{i}\right|-w_{i}\right)\right] / 2,\right.
\end{aligned}
$$

Here $V_{o}$ is a constant to be self-consistently determined, $\kappa$ is $6 \AA^{-1}, i$ labels the atoms in the selected EC participating in electron transfer, $\mathbf{r}_{i}$ is the atom position on that EC, and $w_{i}$ is an element-specific radius. $w_{i}$ amounts to $1.65 \AA$ for $\mathrm{C}$ and $\mathrm{O}$ and $1.25 \AA$ for $\mathrm{H}$. These values are similar to Lennard-Jones radii in simple atomic force fields. A more stringent wavefunction convergence criterion of $10^{-6} \mathrm{eV}$ or smaller is enforced in self-consistent cDFT calculations. The $W(\mathbf{r})$ functional form does not double-count electron density on adjacent atoms and appears pertinent when bond-breaking can occur. Normalized, atomic orbital-based charge projection operators used in the literature $e^{38,45,72}$ may be less applicable for electron transfer coupled to bond-breaking, but they can be tested for the present application in the future.

The total electronic charge on the selected EC is determined by projecting $W(\mathbf{r}) / V_{o}$ on to the DFT electron density. With the $w_{i}$ values mentioned above, unconstrained DFT predicts that a charge-neutral EC molecule adsorbed on the thin $\mathrm{LiAlO}_{2}$ surface (Fig. 4 $4 \mathrm{c}$ ) exhibits a slight $+0.20|e|$ "net charge," while $-0.60 \pm 0.1|e|$ resides on the $\mathrm{EC}^{-}$(Fig. $4 \mathrm{~d} \mathrm{~d}$ ). The adsorbed $\mathrm{EC}^{-}$exhibits a similar $-0.67|e|$ charge on the $\mathrm{LiAlO}_{2}$ oxide slab without any conductive $\mathrm{Li}_{x} \mathrm{C}_{6}$ component (Table I). The non-integer values arise because of residual charge densities at the edge of EC molecules beyond the range of $W(\mathbf{r}) / V_{o}$. (The net spin on $\mathrm{EC}^{-}$is about $0.9|e|$, and is more centered on EC than the net charge.) Increasing $w_{i}$ is ruled out because of the close proximity of adsorbed EC to the surface hydroxyl groups. For example, using larger $w_{i}$ has been found to lead to abstraction of protons from surface hydroxyls. The protons then bind to the negatively charged EC molecule. Such reactions are not seen in unconstrained AIMD simulations and are deemed unphysical. We have therefore defined

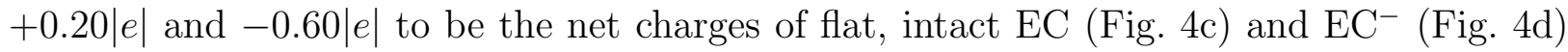
when using self-consistent cDFT calculations to impose charges on the molecule. Increasing 
$\left|V_{o}\right|$ to increase the charge on adsorbed $\mathrm{EC}^{-}$to $-0.80|e|$ is found to yield only a $10 \%$ change on the coupling matrix element $V_{\mathrm{AB}}$, but can increase $\lambda$ by a fraction of an electron volt. The more important parameter, the barrier in Eq. 1, is only affected by $\sim \delta \lambda / 4$ in the harmonic approximation used in this work. In the S.I., the predicted $\lambda$ for adsorbed EC is shown to be comparable to that for EC in liquid EC, computed using cluster calculations, localized orbitals, and a dielectric continuum approximation.

Coupling matrix elements $V_{\mathrm{AB}}$ between the two different adiabatic surfaces (Fig. 1 $\mathrm{k}$ ) are computed using the cDFT formalism for discrete orbital levels $\frac{39}{\underline{9}}$ which is implemented into VASP within the projector-augmented wave formalism. $\frac{68}{\underline{T}}$ The same atomic configuration must be used for both electronic surfaces, and this is chosen to be the optimized atomic configuration where no excess electron resides on the flat, adsorbed EC. $V_{\mathrm{AB}}$ is generally assumed to be relatively independent of atomic positions with the "Franck-Condon" approximation, although molecular orientation dependence has been demonstrated. $\underline{\underline{4}} V_{\mathrm{AB}}$ emerges from the $2 \times 2$ Hamiltonian matrix $H$ connecting the donor $\left(\left|\Phi_{A}\right\rangle\right.$, in our case from unconstrained DFT calculations) and acceptor $\left(\left|\Phi_{B}\right\rangle\right)$ single determinantal wavefunctions $\underline{39}, \underline{72}\left|\Phi_{B}\right\rangle$ features an excess electron on one EC molecule and is generated using cDFT. $H$ contains the overlap matrix element $\left\langle\Phi_{A} \mid \Phi_{B}\right\rangle$ as well as $\left\langle\Phi_{A}\left|\sum_{e} W\left(r_{e}\right)\right| \Phi_{B}\right\rangle$, where $e$ labels all occupied electronic levels $\underline{\underline{39}}$ These calculations are fairly costly and are performed at $\mathrm{T}=0 \mathrm{~K}$ in this work.

This cDFT-based $V_{\mathrm{AB}}$ formulation was originally devised for electron transfer between ground state cDFT donar and acceptor electronic configurations, with the implicit assumption that the relevant density-of-state is discrete. In the limit of non-interacting electrons residing on a metal electrode, this formalism reflects only the top curve on the left side of Fig. 1b and does not reduce to the well-known Fermi Golden Rule formula for tunneling from a continuum of donor states. Consider, in this limit, a band of single-particle energy levels $E$ characterized by a density-of-state $D(E)$ of orbitals $\phi(E)$, Fermi distribution function $f(E)$, Fermi level $E_{\mathrm{F}}$, and an isolated acceptor orbital $\phi_{a}$ with energy $E_{a}$. The Golden Rule rate, associated with multiple level crossings illustrated in Fig. 1 1 , is

$$
k_{\mathrm{GR}} \propto\left\langle\int d E\left|\left\langle\phi(E)|v(E)| \phi_{a}\right\rangle\right|^{2} D(E) f\left(E-E_{\mathrm{F}}\right) \delta\left(E-E_{a}\right)\right\rangle_{\mathbf{R}},
$$

where $v(E)$ is the single-particle coupling matrix element and $\langle O\rangle_{R}$ denotes averaging over nuclear degrees of freedom $R$ on which all quantities implicitly depend. This formula allows 
many-electron acceptor $\left|\Phi_{B^{\prime}}\right\rangle$ states that involve $\phi_{a}$ but not the HOMO of $\left|\Phi_{A}\right\rangle$, which represent electron-hole excitations. $\frac{52}{2}$ In contrast, $\mathrm{cDFT}$ can only generate the electron-acceptor manifold $\left|\Phi_{B^{\prime}}\right\rangle$ which is the ground electronic states within the applied constraint.

To incorporate the effect of Eq. 4, we make the common assumption that $V_{\mathrm{AB}}$ is constant over the relevant range of density-of- state. $\underline{46} \underline{45}$ Then an empirical Golden Rule-like expression can be proposed:

$$
k_{\mathrm{et}}^{\mathrm{GR}}=\sum_{a^{\prime}} f_{a^{\prime}} \frac{\sqrt{\pi}\left|V_{\mathrm{AB}}\right|^{2}}{\hbar \sqrt{\lambda k_{\mathrm{B}} T}} \exp \left[-\frac{\left(\Delta E_{o}+\Delta E_{a^{\prime}}+\lambda\right)^{2}}{4 \lambda k_{\mathrm{B}} T}\right]
$$

Here $\Delta E_{o}$ is used in place of $\Delta G_{o}$ because we ignore entropy changes in $\mathrm{T}=0 \mathrm{~K}$, UHV-setting calculations, $f_{a^{\prime}}$ is the Fermi and/or symmetry weight of Kohn-Sham orbital $a^{\prime}$, and $\Delta E_{a^{\prime}}$ is the difference in energy between the Fermi energy and each Kohn Sham orbital level $a^{\prime}$, $e_{\mathrm{F}}-e_{a^{\prime}}$. $a^{\prime}$ deep within the occupied manifold does not contribute due to the $\Delta E_{a^{\prime}}$ factor. The self-consistent $\Gamma$-point electronic density is used to generate a dense grid of occupied states $\phi_{a^{\prime}}$ using a $1 \times 4 \times 4$ Monkhorst-Pack Brillouin sampling. $\underline{73}$

To converge to the infinite size limit for $e^{-}$transfer to a single EC molecule, the correct approach is not to increase $k$-point sampling, but to increase all spatial dimensions of the model electrode. If the $\mathrm{Li}_{x} \mathrm{C}_{6}$ component of the electrode is doubled in size in any one direction, the orbital donor wavefunction $\phi_{a^{\prime}}$ delocalized over the electrode is scaled down by $\sim 1 / \sqrt{2}$, and $\left|V_{\mathrm{AB}}\right|^{2}$ decreases 2-fold. This underscores the fact that $V_{\mathrm{AB}}$ is not a measurable

quantity in finite-sized electrode models, but changes with the system size. However, the density-of-state $D\left(E_{a^{\prime}}\right)$ increases proportionately with system size, and the sum over all orbital contributions (Eq. 5) should be well-defined in that infinite size limit.

\section{Experimental Details}

Carbon films deposited onto $\mathrm{Cu}$ were used as electrodes to explore the passivating role of the ALD-derived alumina coatings with respect to electrolyte reductive decomposition. Polished AT-cut quartz crystals patterned with $\mathrm{Cu}$ electrodes (9 MHz, Inficion) were used as the base current collector for conducting both voltammetry and gravimetry. $50 \mathrm{~nm}$ thick carbon films were deposited onto these crystals using a pulsed laser deposition method. ${ }^{74,75}$ Conformal alumina coatings were deposited onto both carbon films and bare Cu electrodes at a substrate temperature of $180^{\circ} \mathrm{C}$ using alternate cycles of trimethylaluminum and water 
to produce amorphous $\mathrm{Al}_{2} \mathrm{O}_{3}$ films of either 0.55 or $1.1 \mathrm{~nm}$ thickness $11,76,77$ Cycles of $\mathrm{NO}_{2}$ and TMA pre-exposure were used to ensure the nucleation and growth of a continuous alumina film. ${ }^{78}$ Electrochemical measurements were conducted under argon in a glove box

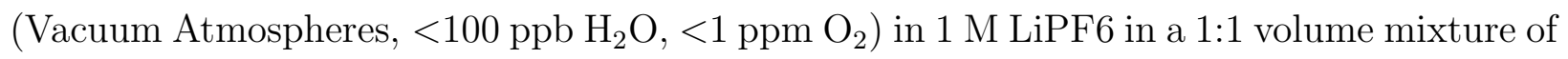
ethylene carbonate and diethylcarbonate (Hoshimoto and Kishida Chemical). A Solartron 1287 potentiostat coupled with a Maxtek RQCM controller were used for simultaneous voltammetric and gravimetric measurements.

\section{RESULTS: ADIABATIC AIMD/DFT PREDICTIONS OF EC/LI(100) REAC- TIONS}

Adiabatic DFT/PBE calculations should be pertinent for the EC/Li(100) interface, where EC and the metallic electrode are in close contact and fast $e^{-}$transfer is expected.

\section{A. Liquid EC on Li (100)}

Liquid EC has been previously predicted to decompose at the $\mathrm{C}=\mathrm{O}$ edges of $\mathrm{LiC}_{6}$ electrodes within $7 \mathrm{ps}$ at $\mathrm{T}=450 \mathrm{~K}$ in AIMD/PBE simulations. ${ }^{21}$ This timescale is used to qualitatively gauge the DFT/PBE predicted reactivity of other surfaces towards liquid EC.

Figure 2b shows that liquid EC decomposes readily on Li (100). Within 15 ps, all 12 EC molecules adjacent to the Li metal, out of $32 \mathrm{EC}$ in the simulation cell, have accepted electrons and decomposed. 11 out of these 12 exhibit two broken $\mathrm{C}_{\mathrm{C}}-\mathrm{O}$ bonds to form $\mathrm{CO}+$ $\mathrm{OC}_{2} \mathrm{H}_{4} \mathrm{O}^{2-} ; 23-26$ only one $\mathrm{EC}$ decomposes in the classic $\mathrm{C}_{2} \mathrm{H}_{4}+\mathrm{CO}_{3}^{2-}$ route hitherto widely accepted in the literature, cleaving both $\mathrm{C}_{\mathrm{E}}-\mathrm{O}$ bonds. $\stackrel{1.2}{ }$ Here $\mathrm{C}_{\mathrm{C}}$ and $\mathrm{C}_{\mathrm{E}}$ are the carbonyl and ethylene carbon atoms, respectively. This finding is consistent with those in Ref. 21, where both $\mathrm{CO}$ and $\mathrm{CO}_{3}^{2-}$ products emerge at the interface between liquid EC and pristine $\mathrm{LiC}_{6}$ with oxidized edge groups. This agreement is significant because, by construction, the models used in Ref. 21 exclude solvent co-intercalation cited in the "3-dimensional" SEI formation pathway. ${ }^{66}$ Nevertheless, fast EC decomposition and identical products are predicted on both pristine graphite and Li metal surfaces, showing that such co-intercalation is not necessary for SEI initiation.

In the $\mathrm{EC} / \mathrm{Li}$ trajectory, the temperature is thermostat at $\mathrm{T}=350 \mathrm{~K}$, not $\mathrm{T}=450 \mathrm{~K}$, 
to avoid melting the solid Li. Despite this, the heat generated by the reactions and the incorporation of CO into the metal slab have caused significant amorphization. In Ref. 15, the initial 200 fs of this trajectory is examined in detail. The bent EC geometry, with the carbonyl $\mathrm{C}=\mathrm{O}$ displacing out of the $\mathrm{EC}$ plane, is shown to be correlated with electron transfer to EC, just like for the isolated $\mathrm{EC}^{-}$in solution (Fig. 11d) ${ }^{12,15}$ This bent geometry plays a critical role in electron transfer and reorganization energy calculations in $\mathrm{LiAlO}_{2}{ }^{-}$ coated surfaces (see below). Our AIMD simulations have shown that $\mathrm{OC}_{2} \mathrm{H}_{4} \mathrm{O}^{2-}$ can react with $2 \mathrm{CO}_{2}$ to form the main SEI organic product ethylene dicarbonate. Whether this product is deposited at the initial stage of SEI growth depends on the availability of $\mathrm{CO}_{2}$ and the solubility of the decomposition fragments..$^{79}$

\section{B. Isolated EC on Li (100)}

Remarkably, even a single EC molecule, in the absence of the liquid environment which stabilizes its ionic breakdown products, still decomposes on $\mathrm{Li}$ (100) surfaces to form CO + $\mathrm{OC}_{2} \mathrm{H}_{4} \mathrm{O}^{2-}$ within picoseconds. (Fig. 2re) This suggests that a simple $\mathrm{T}=0 \mathrm{~K}$ energy profile calculation is relevant to EC decomposition. ${ }^{80}$

Figure 3 a compares the $\mathrm{T}=0 \mathrm{~K}$ energy profiles of the two modes of excess electroninduced EC breakdown on Li metal. They show that cleaving the $\mathrm{C}_{\mathrm{C}^{-}} \mathrm{O}$ bond to form the precursor to carbon monoxide, $\mathrm{OC}_{2} \mathrm{H}_{4} \mathrm{OCO}^{2-}$, is thermodynamically less favorable than the ethylene carbon-oxygen bonds to form $\mathrm{CO}_{3}^{2-}$ and $\mathrm{C}_{2} \mathrm{H}_{4}$ by a substantial $1.53 \mathrm{eV}$. Cleaving the remaining $\mathrm{C}_{\mathrm{C}^{-}} \mathrm{O}$ bond in the $\mathrm{CO}$ route only leads to another $0.16 \mathrm{eV}$ stabilization. The barriers associated with both types of bond-breaking are vanishingly small. Applying the HSE06 truncated hybrid functional, 1,82 which exhibits far less self-interaction errors $32-34$ than PBE, increases the $\mathrm{C}_{\mathrm{C}^{-}} \mathrm{O}$ breaking barrier, but only to $0.16 \mathrm{eV}$ (not shown, but consistent with the similar short time dynamics predicted with the PBE and HSE06 functionals 15 ). This suggests that adiabatic DFT/PBE barrier predictions are reasonably accurate for EC in contact with Li metal. The small barrier explains why both product channels are available in picosecond time scales at explicit liquid EC/electrode interfaces (Fig. 2b, Ref. 15). We speculate that the kinetic prefactor favors the CO-route and makes it the majority product in liquid-solid interface simulations (Fig. 2b). 


\section{Long-range $\mathrm{e}^{-}$transfer formalism is not applicable to $\mathrm{EC} / \mathrm{Li}(100)$}

For $e^{-}$transfer to EC directly adsorbed on uncoated electrode surfaces, the close contact should render the cDFT method for non-adiabatic long-range electron transfer $\underline{\underline{39}} \underline{\underline{43}}$ inapplicable. If one insists on calculating $V_{\mathrm{AB}}$ using cDFT and and the simulation cell described in Table I. $V_{\mathrm{AB}}$ is found to be $0.23 \mathrm{eV}$ for a flat EC adsorbed on Li (Fig. 3b). This large $V_{\mathrm{AB}}$ is consistent with the significant, $56 \%$ overlap between the acceptor and donor many-electron wavefunctions, and should put the system in the adiabatic electron transfer regime - even

with the caveat about the system size dependence of $V_{\mathrm{AB}} \stackrel{\underline{83}}{\text { (For comparison, a theoretical }}$ work on NO molecules adsorbed on $\mathrm{Ag}(111)$, not using cDFT, has also yielded fraction-of-eV $\left.V_{\mathrm{AB}} \stackrel{\underline{48}}{=}\right)$ We conclude that the adiabatic DFT/PBE treatment should suffice in this case.

\section{RESULTS: NON-ADIABATIC ELECTRON TRANSFER TO EC ON OXIDE SURFACES}

This section focuses on a UHV-like model consisting of an isolated EC adsorbed on the lithium-intercalated graphitic carbon strip coated with $\mathrm{LiAlO}_{2}$. A $0.4 \mathrm{~V} / \AA$ electric field is applied. For this model, $e^{-}$tunneling resides in the non-adiabatic regime where cDFT calculations are pertinent. The relevance of this model to the liquid EC/electrode interfacial environment will be clarified below.

\section{A. Two metastable EC charge states on $7 \AA$ thick oxide surface}

The $\mathrm{Li}_{x} \mathrm{C}_{6}$ model with a $7 \AA$ thick $\mathrm{LiAlO}_{2}$ coating proves especially useful for examining the details of electron transfer from the electrode to an adsorbed EC, which either precedes or takes place simultaneously with $\mathrm{EC}^{-}$decomposition. Two (meta)-stable adsorbed EC configurations can be stabilized (Fig. (4). One is a flat, charge-neutral EC coordinated to a surface site (an AlOH group) via its carbonyl oxygen atom (Fig. 4k). Figure 4a depicts the local electronic density-of-state (DOS) for this system. The $\mathrm{Li}_{x} \mathrm{C}_{6}$ region contains partially occupied states near the Fermi level $\left(E_{\mathrm{F}}\right)$. The insulating oxide spans a substantial band gap, although there are surface states in the interface with $\mathrm{Li}_{x} \mathrm{C}_{6}$ that reduce the effective insulating thickness. The highest occupied molecular orbital (HOMO) of $\mathrm{EC}$ is below $-2.5 \mathrm{eV}$ 
while the LUMO lies above $E_{\mathrm{F}}$. This DOS is consistent with a charge-neutral EC weakly interacting with the oxide surface.

The other configuration has an intact $\mathrm{EC}^{-}$which adopts a bent geometry with the $\mathrm{C}=\mathrm{O}$ bond protruding out of the EC plane (Fig. 4 $\mathrm{d}$ ). This is reminiscent of the first stage of liquid EC decomposition on Li (100) surface, where the $e^{-}$-accepting EC adopts a similar bent configuration. $\stackrel{15,84}{ }$ The excess charge on the EC is centered around the carbonyl oxygen atom which is coordinated to two AlOH groups and a Li surface atom. The system exhibits a DOS (Fig. 4b) substantially different from Fig. 4a. The majority spin, highest occupied state of the EC molecule now lies below the Fermi level. The shift in the LUMO upon $e^{-}$addition serves as a caveat against using the LUMO of the neutral molecule as a figure-of-merit in assessing electrochemical reduction tendencies.

The bent $\mathrm{EC}^{-}$is almost iso-energetic with the flat EC. Its slight exothermicity, $\Delta E_{o}=$ $-0.02 \mathrm{eV}$, does not depend on whether the electron transfer is adiabatic or non-adiabatic. It should not be affected by the periodic images imposed by the simulation cell because the dipole correction is applied. ${ }^{71}$ In fact, despite the transfer of an $e^{-}$across a 7 - or $10-\AA$ thick

oxide layer, the overall dipole moment of the simulation cell changes by less than $1.0|e| \AA$, apparently because the electron density in the metallic $\mathrm{Li}_{x} \mathrm{C}_{6}$ strip can rearrange itself to accommodate the electron transfer. The total charge in the simulation cell is conserved in these calculations and the large correction due to periodic boundary conditions for isolated ions in solutions is not needed. $\underline{85}, \underline{86}$ Note that $\Delta E_{o}$ is used in place of $\Delta G_{o}$ because the calculation is performed at $\mathrm{T}=0 \mathrm{~K}$.

\section{B. Non-adiabatic electron transfer on oxide surface}

We apply the cDFT method to calculate $\lambda$ and $V_{\mathrm{AB}}$ required to estimate the electron transfer rate $k_{\text {et }}$ (Eqs. 1 \& 5). We stress that the flat EC absorbed on the oxide coatings is treated using unconstrained DFT/PBE. The highest-occupied orbitals of the $7 \AA$ and $10 \AA$ thick coatings reside in the $\mathrm{Li}_{x} \mathrm{C}_{6}$ region, and exhibit integrated electron densities of less than $10^{-4}$ and $5 \times 10^{-8}|e|$ on the EC molecule, respectively. This shows that the unconstrained DFT method already gives a reasonable description of the neutral EC electronic configuration.

$\lambda$ is computed for the optimized, flat EC geometry adsorbed on the thin $\mathrm{LiAlO}_{2}$ coating 
(i.e. image 0 in Fig. 5a). cDFT imposes an extra electron on the EC molecule. On the $7 \AA$ thick coating, it yields a vertical excitation energy $\Delta E_{\mathrm{vert}}=\lambda+\Delta E_{o}=2.04 \mathrm{eV}$, where $\Delta E_{o}$ is the aforementioned $-0.02 \mathrm{eV}$ offset between donor and acceptor. Alternatively, an electron can be removed from the frozen bent $\mathrm{EC}^{-}$configuration (image 5), which leads to $\lambda^{\prime}+\Delta E_{o}=1.80 \mathrm{eV} . \lambda$ and $\lambda^{\prime}$ agree to within $14 \%$. This is qualitatively consistent with the Marcus theory postulate that the polarization degrees of freedom respond harmonically (Eq. 1), yielding a single reorganization energy that governs electron transfer reactions. $\underline{\underline{31}}$ With $\lambda=2.06 \mathrm{eV}$ for $\mathrm{EC}$ adsorbed on the thin $\mathrm{LiAlO}_{2}$ surface, the non-adiabatic barrier becomes $0.51 \mathrm{eV}$ from a simple Marcus construction (Eq. 1). This barrier is much higher than the $\sim 0.1 \mathrm{eV}$ adiabatic DFT/PBE activation energies for both the $\mathrm{C}_{\mathrm{C}^{-}} \mathrm{O}$ and $\mathrm{C}_{\mathrm{E}^{-}} \mathrm{O}$ bond breaking pathways on this surface (Sec. IVC), and is therefore the rate-limiting step in EC breakdown on the surface of the thin $\mathrm{LiAlO}_{2}$ coating.

In the S.I., an EC with a dielectric approximation of the liquid EC solvent medium is found to exhibit an average of $\lambda=1.76 \mathrm{eV}$, similar to $\mathrm{EC}$ adsorbed on the thin $\mathrm{LiAlO}_{2}$ coating. The co-solvent dimethyl carbonate (DMC) exhibits only slightly smaller $\lambda$ values. Therefore the substantial $\lambda$, large compared to many organic molecules, $\frac{87}{2}$ is intrinsic to out-of-plane bending of the $\mathrm{C}=\mathrm{O}$ group as the carbonyl carbon atom adopts a $s p^{3}$-like geometry to accommodate an $e^{-}$. The S.I. further presents results on vertical excitation energy, $\Delta E_{\text {vert }}=\lambda+\Delta E_{o}$ (Fig. 13), computed in several AIMD snapshots, to suggest that the Arrhenius term in Eq. 1 favors $e^{-}$transfer to EC molecules at the interface over EC in the bulk liquid region. In such AIMD simulations, we are limited to the first choice of $\lambda$, i.e., instantaneously adding an electron to $\mathrm{EC}$, because $\mathrm{EC}^{-}$in liquid $\mathrm{EC}$ can have short lifetimes. $\stackrel{21}{ }$ Hence we will focus on this first choice throughout this work.

As this is a $\mathrm{T}=0 \mathrm{~K}$ calculation in a UHV-like setting, we have simply used the $\left(\Delta E_{o}+\right.$ $\lambda)^{2} /(4 \lambda)$ expression in Eq. 1 as the tunneling barrier, $\underline{39} \underline{42}$ and have not traced out the two adiabatic curves as a function of the energy gap using liquid state potential-of-meanforce simulations $\underline{88}, 89$ We have however checked that, when relaxing $\mathrm{EC}^{-}$frozen in the flat geometry (Fig. 4c) with a constrained charge, it reverts to the stable bent $\mathrm{EC}^{-}$(Fig. 4d) configuration, showing that the cDFT approach puts the system on the correct electronacceptor potential surface. In the future, we plan to perform direct cDFT calculation of the barrier height at $\mathrm{T}=0 \mathrm{~K}$ by simultaneously optimizing the same atomic configuration on both energy surfaces. 


\begin{tabular}{||l|l|l|l||}
\hline \multicolumn{2}{|c|}{ coated electrode } & \multicolumn{2}{c||}{ uncoated electrode } \\
\hline system & work func. & system & work func. \\
\hline thin $\mathrm{LiAlO}_{2}(\mathrm{OH})$ & 2.47 & $\mathrm{Li}(100)$ & 3.05 \\
thick $\mathrm{LiAlO}_{2}(\mathrm{OH})$ & 2.90 & graphite edge & 4.57 \\
thin $\mathrm{LiAlO}_{2}(\mathrm{OLi})$ & 2.25 & $\mathrm{LiAlO}_{2}$ & 5.42 \\
$\mathrm{Al} 2 \mathrm{O} 3(\mathrm{OH})$ & 4.10 & $\mathrm{Al}_{2} \mathrm{O}_{3}(0001)$ & 6.22 \\
\hline
\end{tabular}

TABLE II: Work function of model systems used in this work computed using the PBE functional, in $\mathrm{eV}$. The left column describes the oxide coatings on $\mathrm{Li}_{x} \mathrm{C}_{6}$; the right column refers to work functions of pure crystals. The $\mathrm{Al}_{2} \mathrm{O}_{3}$ (0001) model is Al-terminated. The graphite slab has dangling bonds.

The cDFT coupling matrix element is estimated to be $V_{\mathrm{AB}}=0.022 \mathrm{eV}$ at the flat EC geometry. Fig. 5e depicts the highest-occupied DFT and cDFT orbitals, integrated over the lateral dimensions, for the systems with flat $\mathrm{EC}$ and flat $\mathrm{EC}^{-}$respectively. The overlap between them, $\left\langle\phi_{\mathrm{EC}}^{\mathrm{HOMO}} \mid \phi_{\mathrm{EC}-}^{\mathrm{HOMO}}\right\rangle$, is 0.0125 , or within $2 \%$ of that between the respective determinantal wavefunctions $\left\langle\Phi_{\mathrm{A}} \mid \Phi_{\mathrm{B}}\right\rangle$ (Sec. II) which includes many-electron contributions. Therefore the relaxation of other electrons ("polarization effect") does not strongly influence the overlap integral when using $\Gamma$-point sampling.

This estimate of $V_{\mathrm{AB}}$ does not reflect the classic Fermi Golden rule phenomenology (Sec. III). Applying Eq. 5 to approximately account for the finite density-of-state on the electrode, we obtain a $1.63 \times 10^{4} / \mathrm{s}$ electron transfer rate. Simply using the cDFT definition of $V_{\mathrm{AB}}$ in Eq. 1, which represents a single point integration quadrature, merely underestimates this rate by a factor of 1.68. Using DFT/PBE rather than more accurate but costly hybrid functionals has been known to overestimate $V_{\mathrm{AB}}$ by almost a factor of $10 . \underline{44}$ In the present case, the DFT/PBE underestimation of the band gap of the insulating oxide layer may lead to some overestimation of the electron tunneling rate. Despite the approximations and assumptions involved, this is to our knowledge the first DFT-based estimate of the tunneling rates from an electrode, through an oxide layer, to an adsorbed EC molecule. The value may potentially be compared with UHV measurements. After electron transfer, $\mathrm{EC}^{-}$ decomposes, and the negatively charged EC fragments will most likely complex with $\mathrm{Li}^{+}$ from the electrolyte and be incorporated into the SEI layer on top of the ALD film. 
On the thicker $\mathrm{LiAlO}_{2}$ coating, $\Delta E_{o}=0.77 \mathrm{eV} . \lambda=1.98 \mathrm{eV}$ is predicted in the flat EC geometry. Removing an $e^{-}$from the bent geometry yields $\lambda^{\prime}=1.69 \mathrm{eV}$. The asymmetry is $15 \%$. We again adopt the first choice of $\lambda . V_{\mathrm{AB}}$ is estimated at $0.0128 \mathrm{eV}$, about half that of the $7 \AA$ thick $\mathrm{LiAlO}_{2}$ coating. ${ }^{90}$ As discussed in Sec. IV the thinner coating exhibits substantial surface relaxation which is absent in the $10 \AA$ layer, making a purely thicknessbased comparison of $V_{\mathrm{AB}}$ difficult. Fig. 5d depicts the donor and acceptor Kohn-Sham and cDFT orbitals. The overlap between them, $\left\langle\phi_{\mathrm{EC}} \mid \phi_{\mathrm{EC}-}\right\rangle$, is about 0.004 , a factor of 3 less than that across the $7 \AA$ thick coating. Including the contributions of Eq. 5 , $k_{\text {et }}$ becomes extremely small $\left(2.8 \times 10^{-5} / \mathrm{s}\right)$ due to the larger $\Delta E_{o}$.

The overall $k_{\text {et }}$ is clearly very sensitive to $\Delta E_{o}$ or $\Delta G_{o}$. In UHV settings, $\Delta E_{o}$ depends on both the electric field and surface heterogeneity at atomic lengscales (see below). At electrode/liquid electrolyte interfaces, $k_{\text {et }}$ is a function of the applied voltage as well as the local EC reduction potential via $\Delta G_{o}$ (Eq. 1), which may be a function of the distance from the electrode. Direct measurement of the reduction potential of an intact EC is unavailable because EC decomposition occurs faster than cyclic voltammetry time scales. If one adopts a theoretical $\Delta G_{o}=-0.15 \mathrm{eV}$ for $e^{-}$transfer to intact EC molecules in EC liquid in at $\mathrm{Li}(\mathrm{s}) / \mathrm{Li}^{+}$voltages (S.I.), the predicted initial electron transfer rates $k_{\text {et }}$ through the 7 $\AA$ and $10-\AA$ thick oxide coatings $\left(\sim 1.7 \times 10^{5} / \mathrm{s}\right.$ and $\left.8.3 \times 10^{4} / \mathrm{s}\right)$, will permit electrolyte breakdown, even if we assume that these rates are overestimated by 100 times due to the use of the PBE functional discussed above. Indeed, our gravimetric measurements reveal electrolyte decomposition on the coated electrodes — consistent with ready availability of electrons - albeit in much less quantity than on uncoated electrodes (Sec. V). The electrolyte decomposition product then yields an additional insulating layer that prevents further electron tunneling.

Our main point in this section is not to predict exact $k_{\text {et }}$ values, but to highlight the previously neglected role of the EC reorganization energy $(\lambda)$ on electrode coated with an insulating layer. An immediate implication is that different solvent molecules/salt components may exhibit different $\lambda$ and $e^{-}$-transfer rates. 


\section{DFT/PBE treatment of electron transfer on oxide surface is inadequate}

We next demonstrate that adiabatic DFT/PBE calculations are inadequate when dealing with $e^{-}$tunneling through insulating oxide layers.

The electron transfer barrier strongly depends on whether the $e^{-}$transfer is adiabatic or not, and on the accuracy of the DFT method used. Figure 5a depicts a climbing-image NEB calculation with 4 images along the reaction coordinate linking the flat EC and the bent $\mathrm{EC}^{-}$to examine the $\mathrm{DFT} / \mathrm{PBE}$ adiabatic energy landscape in the $0.4 \mathrm{~V} / \AA$ electric field. DFT/PBE predicts a $0.09 \mathrm{eV}$ barrier associated with electron transfer through the thin $\mathrm{LiAlO}_{2}$ layer.

This small $0.09 \mathrm{eV}$ value gives the strongest indication that DFT/PBE grossly underestimates the $e^{-}$transfer barrier. In classical electron transfer paradigm (Fig. 1a), the parabolic intersection which yields the non-adiabatic barrier in the exponential term in Eq. 1 is expected to differ from an adiabatic prediction of barrier by $V_{\mathrm{AB}}$. Instead, the former is $0.51 \mathrm{eV}$ and the latter is $0.09 \mathrm{eV}$ (Fig 5a); their difference far exceeds $V_{\mathrm{AB}}=0.022 \mathrm{eV}$ before considering system size dependence. The discrepancy is most likely due to the self-interaction error in the DFT/PBE functional, $, 32,34$ a point already alluded to in Ref. 39. The widely used PBE functional, along with others, do not sufficiently penalize configurations where an electron occupies both the electrode and the EC molecule. Indeed, in image 2 of Fig. 5 a, a fractional $-0.2|e|$ charge develops on the EC, which should be considered unphysical for a molecule separated from the electrode by at least $7 \AA$. Hybrid DFT functionals exhibit less self-interaction errors than DFT/PBE, but are currently too costly for computing barriers in interfacial systems of this size..$^{35}$

The $10 \AA$-thick oxide-coated electrode exhibits a monotonic DFT/PBE energy profile for electron transfer. There is no DFT/PBE adiabatic barrier between the flat EC and bent $\mathrm{EC}^{-}$beyond the minimal $0.77 \mathrm{eV}$ mandated by the endothermicity (Fig. 5b), suggesting that the electron tunneling barrier is again severely underestimated. Using the conjugate gradient geometry minimizer in VASP, the bent $\mathrm{EC}^{-}$geometry on this surface is in fact on the verge of instability, about to lose electron density to the electrode and relax to the flat $\mathrm{EC}^{0}$ geometry. Therefore the depicted energy profile actually reflects an optimized geometry subject to a charge constrained via cDFT with a small $V_{o}=-0.2 \mathrm{eV}$. 


\section{Work function and electrochemical potential}

The electron tunneling rate at electrolyte-electrode interfaces depends on the electrochemical potential $(\Phi)$ of the electrode. In the coated graphite model systems, $\Phi$ is not precisely known. Directly calculating $\Phi$ involves averaging the electrostatic potential difference between the conductive (inner) region of the electrodes and a distant point in the bulk liquid beyond the thickness of the electric double layer,, 91 and involves consideration of image charge and surface potential effects. ${ }^{86}, 92-94$ These are beyond the time and length scales of current AIMD simulations. Fortunately, the EC/Li(100) interface mimics immersing freshly prepared Li metal into liquid EC, and reflects an unambigous open-circuit voltage below the threshold at which EC becomes electrochemically decomposed $\left(+0.8 \mathrm{~V}_{\mathrm{vs} . \mathrm{Li}^{+}} / \mathrm{Li}(\mathrm{s})\right)$. This is a major reason $\mathrm{Li}$ is considered in this work.

If we consider the energy of an $e^{-}$in the bulk electrolyte to be a constant, independent of electrode surfaces, the energy for ejecting an electron from different electrodes into the bulk electrolyte will only be shifted by the work function ${ }^{95}$ (where an $e^{-}$goes into vacuum). Thus, we have computed the work functions of coated and uncoated electrode surface and some crystal planes of ALD coating materials (Table II). The -OH and -OLi terminated $\mathrm{LiAlO}_{2}$ coating work functions are within $0.5 \mathrm{eV}$ of the $\mathrm{Li}$ metal value, indicating that similar energies are required to remove an electron from these surfaces. The $\mathrm{Al}_{2} \mathrm{O}_{3}$ coated surface has a much higher work function (Table II), consistent with our observation that $\mathrm{Al}_{2} \mathrm{O}_{3}$ is a more insulating material than $\mathrm{LiAlO}_{2}$ (see below).

Even though our DFT calculations show that placing these oxides in contact with Li metal surfaces leads to immediate Li metal oxidation, we use $\operatorname{Li}(100)$ as a reference because its voltage is similar to that of $\mathrm{LiC}_{6}$. Aligning the work functions of $\mathrm{Li}(100)$ and the oxide materials (Table II), it is clear that the valence and conduction bands of the ALD phase lies below and above the Fermi energy $\left(E_{\mathrm{F}}\right)$ of Li metal, respectively. Electron tunneling from the $\mathrm{Li} E_{\mathrm{F}}$ to the conduction bands of Al-terminated $\mathrm{Al}_{2} \mathrm{O}_{3}(0001)$ and $\mathrm{LiAlO}_{2}(100)$ exhibit $1.43 \mathrm{eV}$ and $1.13 \mathrm{eV}$ offsets (barriers, $\Delta E$ ), respectively.

According to the 1D WKB formula, the tunneling prefactor is

$$
k_{\text {et }} \propto \exp \left(-2 \sqrt{2 m_{e} \Delta E} R / \hbar\right)
$$

where $m_{e}$ is the electron mass. If we take a tunneling transmission probability of $\mathrm{e}^{-40}$ as the limit of vanishing electron tunneling, 3.7nm thick $\mathrm{LiAlO}_{2}$ and $3.2 \mathrm{~nm}$ thick $\mathrm{Al}_{2} \mathrm{O}_{3}$ are required 
to stop total SEI growth using DFT/PBE predicted $\Delta E$. The work function is only one contribution to $\Phi$ and does not contain solvent orientation and electric double layer effects 96 (which should be less important for our inner-shell redox reduction of solvent compared to the classical paradigm of electron transfer to well-solvated outer-shell ions). Nevertheless, it gives a simple guidance for comparing different insulating ALD coating materials. As $e^{-}$ transfer slows down and becomes rate-limiting, the composition of SEI films formed from electrolyte decomposition will likely change. This is because solvent molecules (other than EC), the counter ions $\left(\mathrm{PF}_{6}^{-}\right)$in the salt, and other partially decomposed products may exhibit smaller electron transfer barriers (reorganization energies) and start dominating the product channel.

\section{E. EC bond-breaking on ALD coating after $e^{-}$transfer}

On the $10 \AA$ thick $\mathrm{LiAlO}_{2}$-coated $\mathrm{Li}_{x} \mathrm{C}_{6}$ strip (Fig. 2f), no EC decomposes within 7 ps. The limited duration of the AIMD trajectory does not permit an estimate of the adiabatic AIMD/PBE free energy barrier. While this barrier can be computed using the AIMD/potential-of-mean-force method,,$\frac{17}{1}$ it will be underestimated due to PBE selfinteraction errors and underestimation of the electron tunneling barrier.

However, on the $7 \AA$ thick $\mathrm{LiAlO}_{2}$ layer, a $\mathrm{C}_{\mathrm{C}^{-}} \mathrm{O}$ bond on one EC molecule is spontaneously broken within 1 ps (Fig. 2e), yielding $\mathrm{OCOC}_{2} \mathrm{H}_{4} \mathrm{O}^{-}$, the majority predicted product on Li metal surfaces (Fig. 2b) and a precursor to CO. Here the monovalent anion intermediate is stabilized by hydrogen bond donation from several AlOH groups and by coordination to two surface Li atoms. Since the DFT/PBE method erroneously underestimates the $0.51 \mathrm{eV} e^{-}$tunneling barrier associated with molecular reorganization (Fig. 4a) which precedes bond-breaking, it vastly overestimates the overall bond-breaking rate. Indeed, the EC decomposition timescale predicted with $\mathrm{DFT} / \mathrm{PBE}$ is similar similar to the timescale predicted in the absence of the ALD layer..$^{21}$ This is in disagreement with our experimental measurements which reveals far less solvent decomposition products when an ALD layer is present (Sec. $\mathrm{V})$. Instead, $0.51 \mathrm{eV}$ should be taken as the overall activation energy in these bond-breaking events. With this barrier, the bond-breaking rate should occur in millisecond, not picosecond, timescales at room temperature. Nevertheless, this PBE-based AIMD calculation is valuable because it identifies the most reactive surface site. An EC adsorbed 
at this site is used in the $e^{-}$transfer calculation of the previous section (Fig. 4). Under UHV-like conditions, an isolated EC molecule adsorbed at this site exhibits $<0.05 \mathrm{eV}$ adiabatic DFT/PBE C-O bond-breaking barriers provided a $0.4 \mathrm{~V} / \AA$ electric field is applied (Fig. 6). The qualitative correspondence between adiabatic AIMD/PBE decomposition rate and UHV barrierless reaction is the reason this model is adopted for $e^{-}$transfer studies in Sec. IVB.

Because of its extreme thinness, optimizing the $7 \AA$-thick $\mathrm{LiAlO}_{2}$ film coated on to $\mathrm{Li}_{x} \mathrm{C}_{6}$ has caused $2 \mathrm{Li}$ atoms per surface to migrate outwards (Fig. 2 $\mathrm{k}$ ). These outlying Li coordinate to the surface hydroxyl groups, polarizing them. The EC that undergoes breakdown (Fig. 6d) is indeed hydrogen bonded to an $\mathrm{OH}$ group coordinated to a surface $\mathrm{Li}^{+}$. Such Li migration to the surface does not occur in the thicker $\mathrm{LiAlO}_{2}$ coating. Hence the faster adiabatic AIMD/PBE EC decomposition dynamics on the thin $\mathrm{LiAlO}_{2}$ coating is not just a consequence of oxide thickness, but is partly due to active site chemical specificity. This anomaly may also be the reason the predicted $V_{\mathrm{AB}}$ value does not strongly decrease with increasing the oxide thickness from $7 \AA$ to $10 \AA$, and may further explain the difference in work functions between $\mathrm{Li}_{x} \mathrm{C}_{6}$ coated with $7 \AA$ and $10 \AA$ thick $\mathrm{LiAlO}_{2}$ films (Table II).

The $10 \AA$-thick $\mathrm{LiAlO}_{2}$ coating does not exhibit outward Li atom migration. Here the DFT/PBE bond-breaking barriers of adsorbed EC are not readily deconvolved from $e^{-}$ transfer (S.I.). For simplicity, we consider a model with just one $10 \AA$ thick $\mathrm{LiAlO}_{2}$ layer hydroxylated on both sides (Table I), add one excess $e^{-}$that now always resides on the EC because of the $\mathrm{Li}_{x} \mathrm{C}_{6} e^{-}$sink has been removed, and compute $\mathrm{EC}^{-}$decomposition energetics without applied electric fields. $\mathrm{C}_{\mathrm{E}} \mathrm{O}$ bond-breaking to form $\mathrm{CO}_{3}^{2-}$ precurors remain barrierless and exothermic. However, the $\mathrm{C}_{\mathrm{C}^{-}} \mathrm{O}$ cleavage route to form $\mathrm{CO}$ precurors becomes endothermic and exhibits a $0.71 \mathrm{eV}$ barrier. This indicates a product channel cross-over as the oxide thickness increases and/or the reactivity of the surface site decreases. The expected reaction pathyways transition from a mixture of $\mathrm{C}_{\mathrm{E}^{-}} \mathrm{O}$ and $\mathrm{C}_{\mathrm{C}^{-}} \mathrm{O}$ bond breaking to predominantly $\mathrm{C}_{\mathrm{E}}-\mathrm{O}$ cleavage $\left(\mathrm{CO}_{3}^{2-}\right.$ precursor $)$. While the liquid solvent environment is not included here, we speculate that this finding may be extrapolated to other coating surfaces, including natural SEI films, as the surface sites become less reactive. In the future, we will also examine EC decomposition reactions on $\mathrm{Li}_{2} \mathrm{CO}_{3}$ surfaces to see if similar trends persist on that crystalline material, recently adopted as a theoretical model for organic solvent decomposition SEI film, and the decomposition of other solvent/salt molecules. $\underline{\underline{30}}$ 
We have also conducted AIMD simulations of graphitic anodes coated with $5 \AA$ thick hydroxylated $\mathrm{Al}_{2} \mathrm{O}_{3}$ layers (Fig. 2f). No Li ions reside near the interface region, and no solvent decomposition is observed within $7 \mathrm{ps}$, despite the thinness of the oxide. This emphasizes the importance of surface heterogeneity at atomic lengthscales. Replacing all surface AlOH groups with AlOLi dramatically increases the decomposition rate; this will be discussed in future publications.

\section{EXPERIMENTAL RESULTS}

Figure 7 shows the combined voltammetric and microgravimetric responses of the uncoated and alumina coated PLD carbon films as the electrode potential is decreased to a value slightly above the threshold for $\mathrm{Li}^{+}$intercalation in the carbon. The uncoated carbon electrode (Fig. 7a) exhibits a continuously increasing current response, with several discrete maxima. One maximum reaches a value of $4 \mu \mathrm{A} / \mathrm{cm}^{2}$ with a mass increase of $2 \mu \mathrm{g} / \mathrm{cm}^{2}$ at a potential of $2 \mathrm{~V}$. The other maximum reaches $11 \mu / \mathrm{cm}^{2}$ at a potential of $1 \mathrm{~V}$. The decomposition of the electrolyte and deposition of byproducts at $2 \mathrm{~V}$ is catalyzed by the $\mathrm{Cu}$ substrate, as evidenced by the similar current and mass changes on a control Cu electrode (Fig. 7b), and demonstrate that the carbon films possess porosity and allow electrolyte penetration. As seen in the limiting current and mass profiles of Fig. 7b, electrolyte decomposition results in $\mathrm{Cu}$ passivation beyond $2 \mathrm{~V}$, arguing that the majority of the current and mass changes measured above $2 \mathrm{~V}$ for the porous carbon films (Fig. 7h) are due to electrolyte decomposition on $\mathrm{Cu}$; only the signal below $1 \mathrm{~V}$ is associated with solid electrolyte interphase formation (SEI) on the carbon surface. The porous and therefore higher area carbon surface exhibits a continuous increase in both current and mass uptake as the potential is further reduced from 2 to $0.2 \mathrm{~V}$ and the onset of $\mathrm{Li}^{+}$intercalation is approached. With an approach to $0.2 \mathrm{~V}$, the rate of current change increases substantially over the rate of mass change, signaling a point where $\mathrm{Li}^{+}$intercalation has initiated, where the lighter mass Li (compared to an fragment of ethylene carbonate or diethyl carbonate) accounts for a growing fraction

of the measured current. The possibility exists that current increase could also be related to solvent reduction without mass addition to the surface (soluble byproduct formation), but note that the mass decrease upon reversal of the potential sweep clearly argues for the onset of $\mathrm{Li}^{+}$ion intercalation into the carbon. We note that the scan rate of $1 \mathrm{mV} / \mathrm{s}$ is sufficiently 
fast to produce only modest extraction of Li during this reverse partial half cycle.

The alumina coating acts as a kinetic barrier to prevent electron transfer to the organic carbonate molecules of the electrolyte. Figures $7 \mathrm{c}$, d show the response of a 0.55 and $1.1 \mathrm{~nm}$ thick coated carbon films to the onset of electrolyte reductive decomposition. Comparison of the uncoated ( $\mathrm{Cu}$ subtracted) and coated carbon films shows that a higher overpotential is required to drive solvent decomposition and a lower quantity of mass addition takes place with the alumina coating present. A Cu current and mass uptake response is eliminated for these coated electrodes because the alumina nucleating agent and film precursors fully penetrate the porous carbon, conformally coating both the carbon network and the underlying exposed regions of the $\mathrm{Cu}$ substrate. The onset for significant current density and mass increase occurs at approximately $1.2 \mathrm{~V}$ and $0.8 \mathrm{~V}$ for the $0.55 \mathrm{~nm}$ and $1.1 \mathrm{~nm}$ alumina coatings, compared to $1.5 \mathrm{~V}$ for the uncoated carbon. Mass increases measured at $0.8 \mathrm{~V}$ are $6,1.3$ and $0.5 \mu \mathrm{g} / \mathrm{cm}^{2}$ for the uncoated ( $\mathrm{Cu}$ subtracted), $0.55 \mathrm{~nm}$ and $1.1 \mathrm{~nm}$ alumina sample, respectively. The greater overpotential and reduced mass uptake of the $1.1 \mathrm{~nm}$ coating relative to the thinner $0.55 \mathrm{~nm}$ coating argue that the thicker film provides a more effective kinetic barrier for reducing the extent of both reductive solvent decomposition and byproduct deposition on the electrode. The thicker alumina film would be expected to present a lower electron tunneling rate resulting in a slower rate of solvent decomposition and retarded SEI formation. The fact that mass addition is observed in the presence of these alumina coatings is a clear indicator that alumina serves to retard and limit the extent of but does not prevent electrolyte reduction and resulting byproduct film formation.

\section{CONCLUSIONS}

In this work, we compare EC decomposition on Li metal and on models of oxide-coated electrodes. The latter mimics recent experimental work using ALD technique to passivate anodes. This ALD strategy carries significant technological promise, $\frac{6-11}{-11}$ and it also provides an ideal robust platform for theoretical and experimental study of passivating mechanisms. These two systems represent two electron transfer regimes.

On pristine Li (100) surfaces, liquid EC and even isolated adsorbed EC molecules are predicted to undergo decomposition in picosecond time scales. CO is the dominant product from EC, possibly because of favorable kinetic prefactors, even though both the CO and 
$\mathrm{CO}_{3}^{2-}$ reaction pathways are almost barrierless and the $\mathrm{CO}_{3}^{2-}$ product is more thermodynamically stable. EC molecules and the electrode are in close contact and strongly coupled. Adiabatic DFT/PBE and AIMD/PBE simulations should be accurate in this regime.

In contrast, electron transfer through an oxide layer should be slow compared to nuclear motion. We find evidence that tunneling through even a $7 \AA$ thick oxide layer belongs to the non-adiabatic regime. Applying constrained DFT (cDFT) calculations, such thin coatings are found to slow down $e^{-}$transfer because the solvent reorganization energy $\lambda$ now figures prominently in electron tunneling through the oxide. $\lambda$, largely neglected in previous studies of electrolyte decomposition in batteries, is estimated to be $\sim 2 \mathrm{eV}$ for adsorbed EC molecules in ultra-high vacuum-like conditions. This translates into a $\sim 0.5 \mathrm{eV}$ electron tunneling barrier within the harmonic approximation when the $e^{-}$transfer free energy change is small.

cDFT calculations show that the $7 \AA$ - and $10 \AA$-thick $\mathrm{LiAlO}_{2}$ coated $\mathrm{Li}_{x} \mathrm{C}_{6}$ exhibit electron transfer rates of $\sim 10^{5} / \mathrm{s}$ at the $\mathrm{Li}^{+} / \mathrm{Li}(\mathrm{s})$ applied voltage. The predicted $e^{-}$transfer rate is not free of ambiguities and assumptions, and is of order-of-magnitude utility; further fundamental research is needed for a more rigorous treatment. Despite this caveat, this work respresents the first first-principles estimate of the $e^{-}$tunneling rate between an electrode and an EC molecule across an insulating oxide layer. Such predictions are critical for understanding ALD-hindered SEI growth in lithium ion batteries.

The overall electron transfer rate (Eq. 10r Eq. (5)) also depends on the offset $\Delta G_{o}$ between $e^{-}$donor and acceptor species. $\Delta G_{o}$ in turn depends on the applied voltage. AIMD estimates of $\Delta G_{o}$ in an explicit liquid solvent environment is currently lacking, and we have relied on dielectric continuum treatments of the liquid environment. Nevertheless, our analysis yields useful insights. With any reasonable estimate of $\Delta G_{o}$, the electron transfer rate to EC at the surface is predicted to be faster than $1 / \mathrm{s}$, and solvent breakdown on the ALD oxide is expected. This is confirmed by our gravimetric measurements on ALD-coated anodes, although the amount of solvent decomposition product is significantly less than that on uncoated graphite electrodes.

In the case of oxide-coated electrodes, AIMD/PBE and DFT/PBE calculations without electronic constraints vastly underestimate the electron transfer barrier. The reason is most likely the self-interaction error, which unphysically favors a split electron partially localized on the EC and partially delocalized on the electrode. This defect exists in many 
DFT functionals and has been known to yield errors in when a molecular is split into two fragments. $\underline{32}$ As a result, direct AIMD/PBE simulations overestimate EC decomposition rates at oxide-coated electrode surfaces by many orders of magnitude. However, AIMD/PBE and DFT/PBE calculations still provide a wealth of information about structure and relative energetics, and they form the basis of Marcus theory considerations and non-adiabatic electron transfer studies which are key aspects of this work.

Taking advantage of the qualitative correspondence between AIMD liquid state reaction rates and ultra-high vacuum-like DFT calculations of barrier heights at $\mathrm{T}=0 \mathrm{~K}$ in an electric field, we have applied calculations in UHV-like settings to suggest that the dominant product may shift from a mixture of $\mathrm{CO}$ and $\mathrm{CO}_{3}^{2-}$ to mainly $\mathrm{CO}_{3}^{2-}$ as the binding of ionic decomposition products becomes less favorable (e.g., on thicker oxide coatings). This prediction may be transferrable to natural SEI films arising entirely from electrolyte decomposition. Atomic-scale surface heterogeneity is found to affect EC decomposition, with $\mathrm{Li}^{+}$ions at the surface playing a facilitating or "catalytic" role. Our work paves the way for novel future experimental studies in UHV settings.

\section{Acknowledgement}

We thank John Sullivan, Steve Harris, Na Sai, Anatole von Lilienfeld, and David Rogers for useful discussions, Michael Siegal and Donald Overmyer for the nanoporous carbon samples, and Xingcheng Xiao for sharing Ref. 60 prior to publication. Sandia National Laboratories is a multiprogram laboratory managed and operated by Sandia Corporation, a wholly owned subsidiary of Lockheed Martin Corporation, for the U.S. Deparment of Energy's National Nuclear Security Administration under contract DE-AC04-94AL85000. KL (apart from the work on lithium metal modeling) was supported by Nanostructures for Electrical Energy Storage (NEES), an Energy Frontier Research Center funded by the U.S. Department of Energy, Office of Science, Office of Basic Energy Sciences under Award Number DESC0001160. 


\section{Supporting Information Available}

Further information are available regarding reorganization energies of $\mathrm{EC}$ in bulk liquid, vertical excitation energies of $\mathrm{EC}$ at $\mathrm{LiAlO}_{2}$ /liquid $\mathrm{EC}$ interfaces, DFT/PBE predictions of adiabatic bond-breaking barriers, and discussions of possible $\mathrm{LiAlO}_{2}$ stoichiometry on ALD

$\mathrm{Al}_{2} \mathrm{O}_{3}$ coatings upon cycling power. This information is available free of charge via the Internet at http://pubs.acs.org/. 
1 Advances in lithium-ion batteries, edited by van Schalkwijk, W.A. \& Scrosati, B. (Kluwer, New York, 2002).

2 Lithium-ion batteries: solid-electrolyte interphase, edited by Wang Y.; Balbuena P.B. (Imperial College, London, 2004).

3 Xu, K. Chem. Rev. 2004, 104, 4304.

4 Aurbach, D.; Ein-Eli, Y.; Chusid, O.; Carmeli, Y.; Babai, M.; Yamin, H. J. Electrochem. Soc. 1994, 141, 603; Aurbach, D.; Markovsky, B.; Shechter, A.; Ein-Eli, Y; Cohen, H. ibid. 1996, 143,3809 .

5 Arora, P.; White, R.E.; Doyle, M. J. Electrochem. Soc. 1998, 145, 3647.

6 Riley, L.A.; Cavanagh, A.S.; George, S.M.; Lee, S.-H.; Dillon, A.C. Electrochem. Solid State Lett. 2011, 14, A29 (2011).

7 Riley, L.A.; Cavanagh, A.S.; George, S.M.; Jung, Y.S.; Yan, Y.F.; Lee, S.H.; Dillon, A.C. ChemPhysChem 2010, 11, 2124.

8 Jung, Y.S.; Cavanagh, A.S.; Dillon, A.C.; Groner, M.D.; George, S.M.; Lee, S.-H. J. Electrochem. Soc. 2010, 15\%. A75.

9 Scott, I.D.; Jung, Y.S.; Cavanagh, A.S.; Yan, Y.; Dillon, A.C.; George, S.M.; Lee, S.-H. Nano Lett. 2011, 11, 414.

10 Riley, L.A.; Van Atta, S.; Cavanagh, A.S.; Yan, Y.F.; George, S.M.; Liu, P.; Dillon, A.C.; Lee, S.-H. J. Power Sources 2011, 196, 3317

11 Jung, Y.S.; Cavanagh, A.S.; Leah, R.A.; Kang, S.H.; Dillon, A.C.; Groner, M.D.; George, S.M.; Lee, Y.H. Adv. Mater. 2010, 22, 2172.

12 Wang, Y.; Nakamura, S.; Ue, M.; Balbuena, P.B. J. Am. Chem. Soc. 2001, 123, 11708.

13 Han, Y.K.; Lee, S.U. Theor. Chem. Acc. 2004, 112, 106.

14 Vollmer, J.M.; Curtiss, L.A.; Vissers, D.R.; Amine, K. J. Electrochem. Soc., 2004, 151, A178.

15 Yu, J.M.; Balbuena, P.B.; Budzien, J.L.; Leung, K. J. Electrochem. Soc. 2011, 158, A400.

16 Nair, N.N.; Schreiner, E.; Marx D. J. Am. Chem. Soc. 2006, 128, 13815.

17 Leung, K.; Nielsen, I.M.B.; Criscenti, L.J. J. Am. Chem. Soc. 2009, 131, 18358.

18 Cheng, J.; Sprik, M. J. Chem. Theor. Comp. 2010, 6, 880.

19 Sulpizi, M.; Sprik, M. Phys. Chem. Chem. Phys. 2008, 10, 5238. 
20 Zipoli, F.; Car, R.; Cohen, M.H.; Selloni, A. J. Am. Chem. Soc. 2010, 132, 8593.

21 Leung, K.; Budzien, J.L. Phys. Chem. Chem. Phys. 2010, 12, 6583.

22 For AIMD simulations of electrolytes, see also: Del Popolo, M.G.; Lynden-Bell, R.M.; Kahanoff, J. J. Phys. Chem. B 2005, 109, 5895; Ganesh, P.; Jiang, D.; Kent, P.R.C. J. Phys. Chem. B 2011, 115, 3085;

23 Onuki, M.; Kinoshita, S.; Sakata, Y.; Yanagidate, M.; Otake, Y.; Ue, M.; Deguchi, M. J. Electrochem. Soc. 2008, 155, A794.

24 Ota, H.; Sakata, Y.; Inoue, A.; Yamaguchi, S. J. Electrochem. Soc. 2004, 151, A1659.

25 Gachot, G.; Ribiere, P.; Mathiron, D.; Grugeon, S.; Armand, M.; Leriche, J.-B.; Pilard, S.; Laruelle, S. Anal. Chem. 2011, 83, 478.

26 Marom, R.; Haik, O; Aurbach, D.; Halalay, I.C. J. Electrochem. Soc. 2010, 157, A972.

27 Ein-Eli, Y. Solid-State Lett. 1999, 2, 212; Aurbach, D.; Teller, H.; Levi, E. J. Electrochem. Soc. 2002, 149, A1255.

28 Peled, E.; Menachem, C.; Bar-Tow, D.; Melman, A. J. Electrochem. Soc., 1996, 143, L4.

29 Leong, M.; Doris, B.; Kedzierski, J.; Rim, K.; Yang, M. Science 2004, 306, 2057.

30 To partially circumvent this problem, $\mathrm{Li}_{2} \mathrm{CO}_{3}$ crystals have been adopted as SEI models. See Iddir, H.; Curtiss, L.A. J. Phys. Chem. C 2010, 114, 20903.

31 Marcus, R.A. Rev. Mod. Phys. 1993, 65, 599.

32 Cohen, A.J.; Mori-Sanchez, P.; Yang W.T. Science 2008, 321, 792.

33 Mori-Sanchez, P.; Cohen, A.J.; Yang W.T. Phys. Rev. Lett. 2008, 100, 146401.

34 Sai, N.; Barbara, P.; Leung, K. Phys. Rev. Lett. 2011, 106, 226403.

35 Hybrid DFT functionals have been applied to frozen interfacial geometries comprising hundreds of atoms, but geometry optimization is much more costly there. See, e.g., Sai, N.; Leung, K.; Chelikowsky, J.R, Phys. Rev. B 2011, 83, 121309.

36 An oft-cited example of self-interaction error manifests itself in the dissociation of $\mathrm{H}_{2}^{+}$in the gas phase. The lone electron should reside on one of the H's as the two H nuclei become widely separated, but many DFT functionals predict half an electron on each.

37 Prezhdo, O.V.; Kindt, J.T.; Tully, J.C. J. Chem. Phys. 1999, 111, 7818.

38 Van Voorhis, T.; Kowalczyk, T.; Kaduk, B.; Wang, L.P.; Cheng, C.L.; Wu, Q. Annu. Rev. Phys. Chem. 2010, 61, 149.

39 Wu, Q.; van Voorhis, T. J. Phys. Chem. A 2006, 110, 9212. 
40 Newton, M.D. Chem. Rev. 1991, 91, 767.

41 Kuznetsov, A.M.; Ulstrup J. Electron transfer in chemistry and biology: an introduction to the theory (Riley, Chichester, 1999) pp. 214-217.

42 Deskins, N.A.; Dupuis, M. Phys. Rev. B 2007, 75, 195212; our notation follows this work.

$43 \mathrm{Wu}, \mathrm{Q}$; van Voorhis, T. Phys. Rev. A 2005. 72, 024502.

44 Oberhofer, H.; Blumberger, J. J. Chem. Phys. 2010, 133, 244105.

45 Sit, P.H.L.; Cococcioni, M.; Marzari, N. Phys. Rev. Lett. 2006, 97, 028303.

46 Behler, J.; Reuter, K.; Scheffler M. Phys. Rev. B 2008, 77, 115421.

47 Rogal, J.; Reuter, K.; Scheffler, M. Phys. Rev. B 2007, 75, 205433.

48 Sharani, R.; Shenvi, N.A.; Tully, J.C. J. Chem. Phys. 2009, 130, 174716.

49 Dogonadze, R.R.; Kuznetsov, A.M.; Levich, V.G. Electrochim. Acta, 1968, 13, 1025.

50 Schmickler, W. J. Electronanal. Chem. 1986, 204, 31.

51 Halley, J.W.; Hautman J. Phys. Rev. B 1988, 38, 11704.

52 Sebastian, K.L. J. Chem. Phys. 1989, 90, 5056.

53 Straus, J.B.; Calhoun, A.; Voth. G.A. J. Chem. Phys. 1995, 102, 529.

54 Calhoun, A.; Koper, M.T.M.; G.A. Voth. Chem. Phys. Lett. 1999, 305, 94.

55 Tanaka, S.; Hsu C.P. J. Chem. Phys. 1999, 111, 11117.

56 Kurth, S.; Stefanucci, G.; Almbladh, C.-O.; Rubio, A.; Gross, E.K.U. Phys. Rev. B 2005, 72 035308 .

57 Hu, C.; Hirai, H.; Sugino, O. J. Chem Phys. 2007, 127, 0641103.

58 Tavernelli, I.; Curchod, B.F.E.; Rothlisberger, U. J. Chem. Phys. 2009, 131, 196101.

59 Xu, K.; Xu, J.; Deng, P.Z.; Zhou, Y.Z.; Zhou, G.Q.; Qiu, R.S.; Fang, Z.J. J. Crystal Growth 1998, 193, 127.

60 Xiao, X.C., Lu, P.; Ahn, D.J. Adv. Mater. DOI:10.1002/adma.201101915.

61 Wyckoff, R.W.G. Crystal Structures Vol. 2, 2nd ed. (Interscience, New York, 1965) pp. 305.

62 Marezio, M. Acta Cryst. 1965, 19, 396.

63 Ouyang, C.Y.; Sljivancanin, Z.; Baldereschi, A. Phys. Rev. B 2009, 79, 235410.

64 Bridel, J.-S.; Grugeon, S.; Laruelle, S.; Hassoun, J.; Reale, P.; Scrosati, B.; Tarason, J.-M. J. Power Sources 2010, 195, 2036.

65 The reduction potential of $\mathrm{Li}^{+}(\mathrm{EC}) \rightarrow \mathrm{Li}(\mathrm{s})$ should be similar to that of $\mathrm{Li}^{+}(\mathrm{aq}) \rightarrow \mathrm{Li}(\mathrm{s})$ because the $\mathrm{Li}^{+}$solvation free energy in water and in EC are predicted to be similar, differing by at 
most 0.2 eV. See Johansson, P.; Jacobsson, P. Solid State Ionics 2006, 177 , 2691.

66 Besenhard, J.O.; Winter, M.; Yang, J.; Biberacher, W. J. Power Sources 1995, 54, 228.

67 Kresse, G.; Furthmüller, J. Phys. Rev. B 1996, 54, 11169, Comput. Mater. Sci. 1996, 6, 15.

68 Kresse G.; Joubert, D. Phys. Rev. B 1999, 59, 1758.

69 Perdew, J.P., Burke, K.; Ernzerhof, .M. Phys. Rev. Lett. 1996, 77, 3865.

70 Henkelman, G.; Uberuaga, B.P.; Jonsson, H. J. Chem. Phys. 2000, 113, 9901.

71 Neugebauer, J.; Scheffler, M. Phys. Rev. B 1992, 46, 16067.

72 Oberhofer, H.; Blumberger, J. J. Chem. Phys. 2009, 131, 064101.

73 Our treatment of $k$-point sampling is qualitatively similar to: Schultz, P.A. Phys. Rev. Lett. 2006, 96, 246401. The cDFT orbitals in Figs. $5 \mathrm{c} \& \mathrm{~d}$ are isolated in energy from other orbitals. If a truly continuum of electronic states are used, cDFT-based $V_{\mathrm{AB}}$ calculations would be difficult to implement.

74 Siegal, M.P.; Yelton, W.G.; Overmyer, D.L.; Provencio, P.P. Langmuir 2004, 20, 1194.

75 Siegal, M.P.; Overmyer, D.L.; Kottenstette, R.J.; Tallant, D.R.; Yelton, W.G. Appl. Phys. Lett. 2002, 80, 3940 .

76 Dillon, A.C.; Ott, A.W.; Way, J.D.; George, S.M. Sur. Sci. 1995, 322, 230.

77 Ott, A.W.; Klaus, J.W.; Johnson, J.M.; George, S.M. Thin Solid Films 1997, 292, 135.

78 Cavanagh, A.S.; Wilson, C.A.; Weimer, A.W.; George, S.M. Nanotechnology 2009, 20, 255602.

79 Tasaki, K.; Harris, S.J. J. Phys. Chem. C 2010, 114, 8076.

80 We stress that liquid EC does not exist at $\mathrm{T}=0 \mathrm{~K}$; only single adsorbed molecule can be investigated this way.

81 Heyd, J.; Scuseria, G.E.; Ernzerhof, M. J. Chem. Phys. 2003, 118, 8207; Heyd, J.; Scuseria, G.E.; Ernzerhof, M. J. Chem. Phys. 2006, 124, 219906; ibid, Vydrov, O.A.; Heyd, J.; Krukau, A.V.; Scuseria, G.E. 2006, 125, 074106.

82 HSE06 calculations are performed using VASP 5.2. See Paier, J.; Marsman, M.; Kresse, G. J. Chem. Phys. 2007, 127, 024103.

83 For $V_{\mathrm{AB}}=0.23 \mathrm{eV}$ and $\lambda \approx 2 \mathrm{eV}$ (predicted for EC adsorbed on electrode surfaces, Sec. IVB), the "transmission coefficient" $\kappa=2 P_{\mathrm{LZ}} /\left(1+P_{\mathrm{LZ}}\right)=14.4$, where $P_{\mathrm{LZ}}$ is the Landau-Zenner factor (see, e.g., Ref. 42). $\kappa>1$ is associated with the adiabatic regime.

84 A single $\mathrm{EC}^{-}$embedded in dielectric continuum approximation of the liquid environment also exhibits such geometry. $\frac{12}{2}$ See also the S.I. 
85 VandeVondele, J.; Ayala, R.; Sulpizi, M.; Sprik, M. J. Electroanal. Chem. 2007, 607, 113; Tateyama, Y.; Blumberger, J.; Ohno, T.; Sprik, M. J. Chem. Phys. 2007, 126, 204506; Adriaanse, C.; Sulpizi, M.; VandeVondele, J.; Sprik, M. J. Am. Chem. Soc. 2009, 131, 6046.

86 Cheng, J.; Sulpizi, M.; Sprik, M. J. Chem. Phys. 2009, 131, 154504.

87 Coropceanu, V.; Cornil, J.; da Silva Filho, D.A.; Olivier, Y.; Silbey, R.; Bredas, J.L. Chem. Rev. 2007, 10\%, 926.

88 Zeng, X.C; Hu, H,; Hu, X.Q.; Cohen, A.J.; Yang, W.T. J. Chem. Phys. 2008, 128, 124510.

89 Moens, J.; Seidel, R.; Geerlings, P.; Faubel, M.; Winter, B.; Blumberger, J. J. Phys. Chem. B, 2010, 114, 9173 .

90 The cDFT ground state for $\mathrm{EC}^{-}$adsorbed on the thick $\mathrm{LiAlO}_{2}$-coated electrode is a spin triplet. To compute $V_{\mathrm{AB}}$, which requires the overlap integral $\left\langle\Phi_{A} \mid \Phi_{B}\right\rangle$, a triplet donor state $\mid \Phi_{A}\langle$ is used. If we forcibly move the highest occupied orbital in the $\mathrm{EC}^{-}\left|\Phi_{B}\right\rangle$ into the minority spin channel to overlap with the original singlet $\left|\Phi_{A}\right\rangle$, the resulting $V_{\mathrm{AB}}$ is larger by $73 \%$. This may be taken as an estimate of the uncertainty.

91 Price, D.L.; Halley, J.W. J. Chem. Phys. 1995, 102, 6603.

92 Lozovoi, A.Y.; Alavi, A.; Kohanoff, J.; Lynden-Bell, R.M. J. Chem. Phys. 2001, 115, 1661.

93 Pratt, L.R. J. Phys. Chem. 1992, 96, 25.

94 Leung, K. J. Phys. Chem. Lett. 2010, 1, 496.

95 Lang. N.D.; Kohn, W. Phys. Rev. 1971, 3, 1215.

96 For another discussion of the relationship between $\Phi$ and the work function, see Trasatti, S. Sur. Sci. 1995, 335, 1. We stress that our focus of electron transfer to solvent molecules differs from the classical paradigm of outer-shell electron transfer to ions. Note that the experimental definition of "open circuit voltage" includes interfacial contributions, unlike some first principles theoretical formulations. 


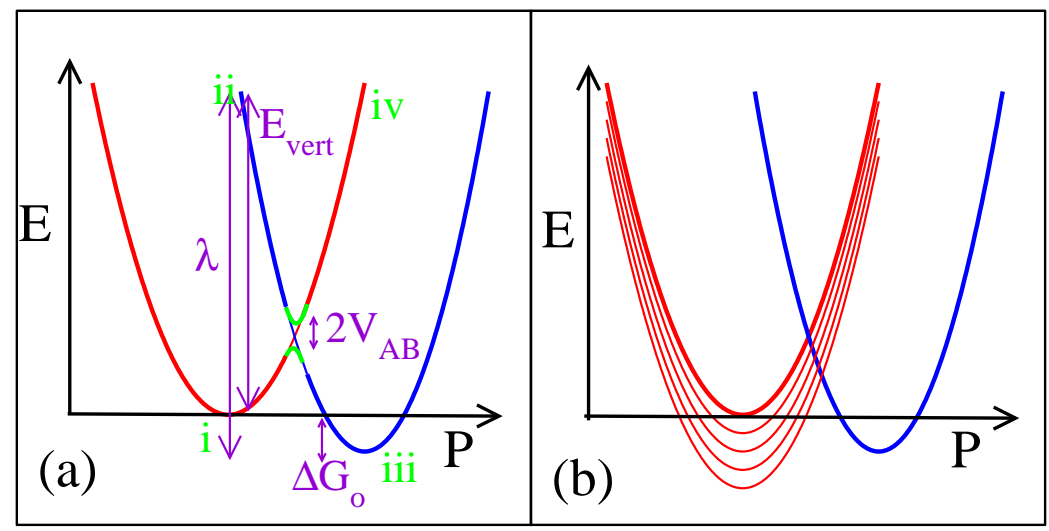

(b)
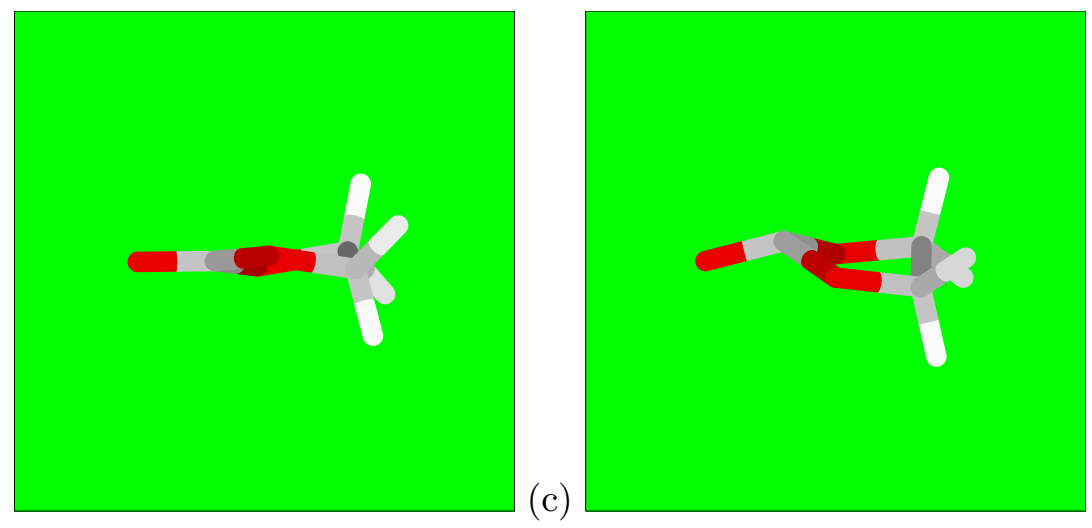

FIG. 1: (a) Schematic of electron transfer between isolated orbitals. The red and blue represent the diabatic potential energy surfaces of $e^{-}$donor and acceptor as a function of the generalized polarization $(P)$ degree of freedom. The green segments represent adiabatic processes with the non-crossing surfaces split by $2 V_{\mathrm{AB}} . \Delta G_{O}$ is the reaction free energy and $\lambda$ is the reorganization energy. The green roman numbers denote (i) flat EC; (ii) flat $\mathrm{EC}^{-}$; (iii) bent $\mathrm{EC}^{-}$; (iv) bent EC. (b) Non-adiabatic $e^{-}$transfer form a metallic electrode. The thick upper red line represents the Fermi level, and is the primary donor orbital within cDFT calculations. $e^{-}$can also transfer from the continuum of electrode donor states below the Fermi level, depicted as thin red lines, to the acceptor orbital, with however increased non-adiabatic barriers (crossing points between blue and red curves). (c) \& (d) Flat and bent EC molecules, respectively. Red, grey, and white refer to O, $\mathrm{C}$, and $\mathrm{H}$ atoms. 
(a)

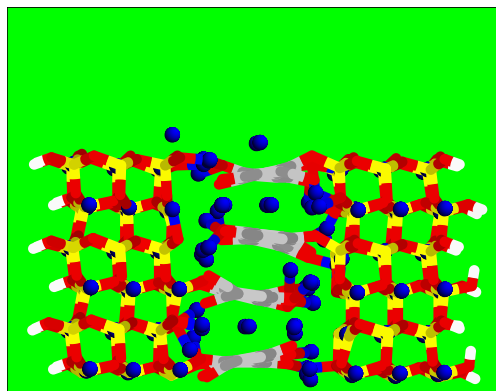

(d)

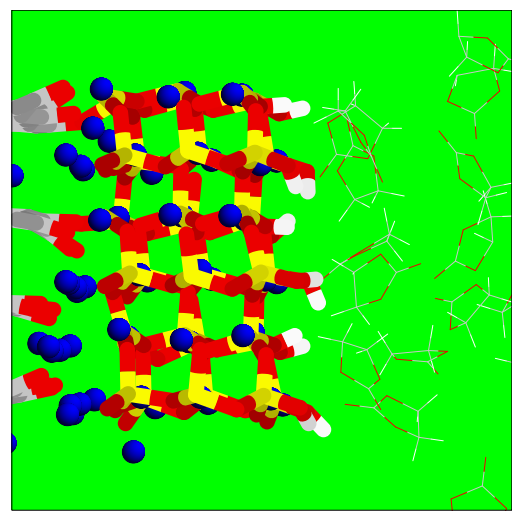

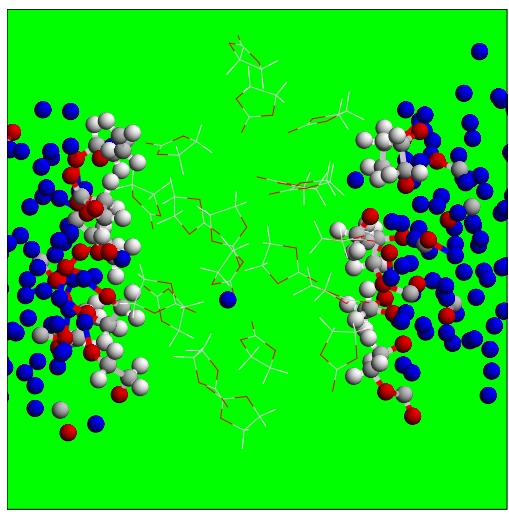

(b)

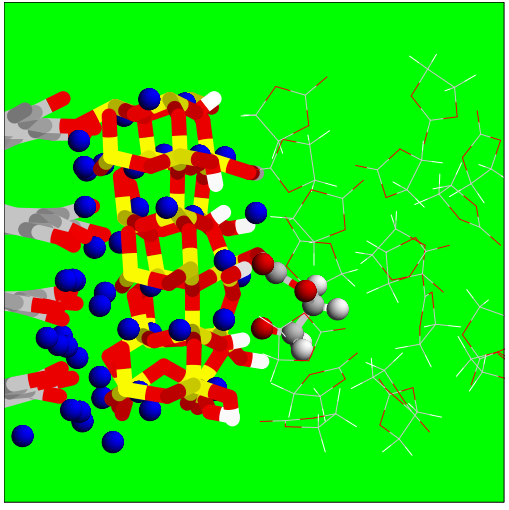

(c)

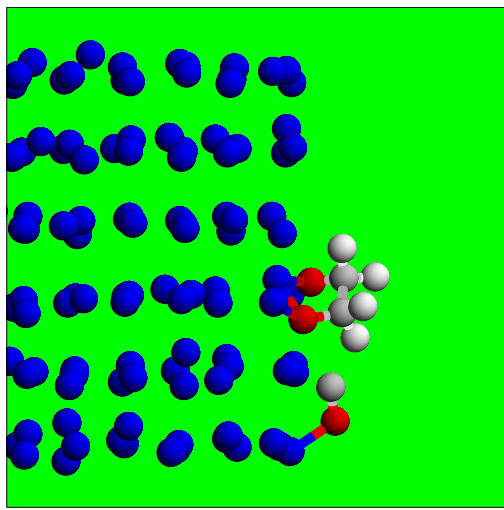

(f)

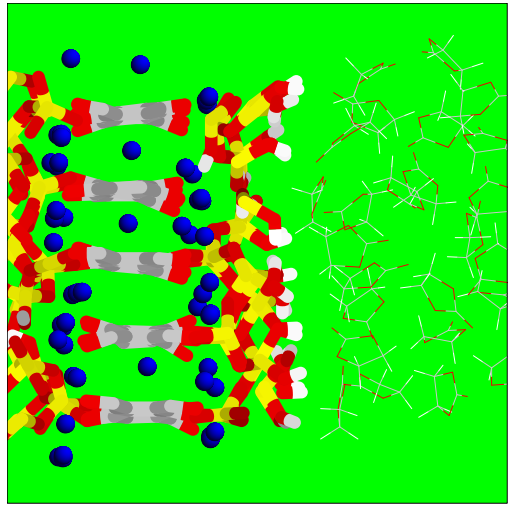

FIG. 2: (a) Model electrode system with a narrow $\mathrm{LiC}_{6}$ strip decorated with $\mathrm{C}=\mathrm{O}$ edges and coated with a $10 \AA$ thick $\mathrm{LiAlO}_{2}$ layers terminated by hydroxyl groups. (b) A snapshot 15 ps into an AIMD trajectory of $32 \mathrm{EC}$ molecules confined between Li metal (100) slabs conducted at T=350 K. Li and decomposed EC are depicted as ball-and-stick models, intact EC as wireframes. (c) An isolated, decomposed EC on Li surface at $\mathrm{T}=350 \mathrm{~K}$, after a 7 ps AIMD simulation. (d) No decomposition on $10 \AA$ thick $\mathrm{LiAlO}_{2}$-coating after 7 ps. (e) One EC decomposed on $7 \AA$ thick $\mathrm{LiAlO}_{2}$-coated surface, $\mathrm{T}=450 \mathrm{~K}$, after 7 ps. (f) No decomposition on $5 \AA$ thick, hydroxylated $\mathrm{Al}_{2} \mathrm{O}_{3}$-coating after 7 ps. Yellow, grey, red, blue, and white depict Al, C, O, Li, H atoms, respectively. 

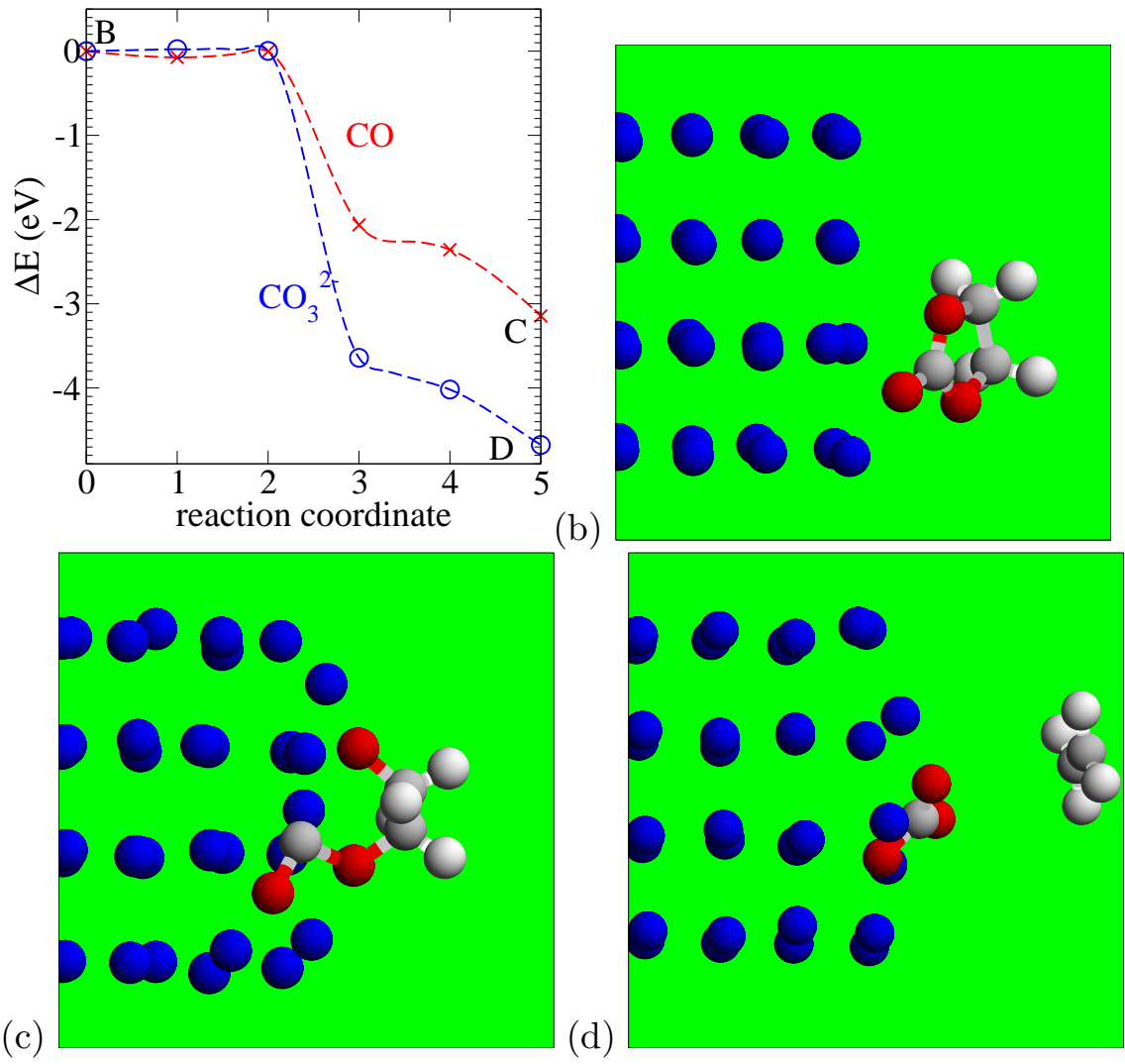

(b)

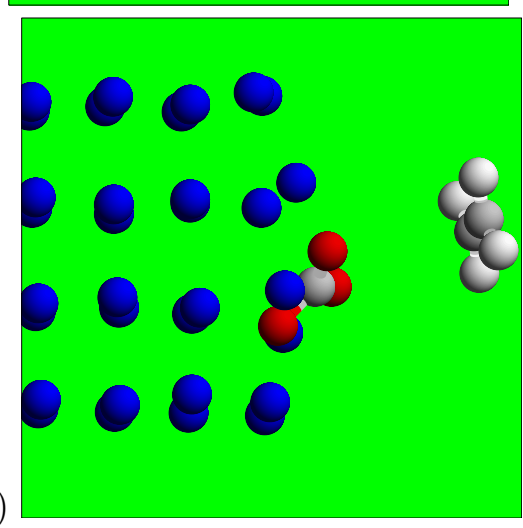

FIG. 3: (a) Static, nudged elastic band (NEB) calculations of energy barriers associated with 2 modes of EC breakdown, producing $\mathrm{CO}$ or $\mathrm{CO}_{3}^{2-}$, at $\mathrm{T}=0 \mathrm{~K}$. Points $\mathrm{B}, \mathrm{C}, \& \mathrm{D}$ correspond to panels (b)-(d). (b) Intact EC on $\mathrm{Li}(100)$. (c) EC partially decomposed into $\mathrm{OCOC}_{2} \mathrm{H}_{4} \mathrm{O}$, precursor to $\mathrm{CO}$ and $\mathrm{OC}_{2} \mathrm{H}_{4} \mathrm{O}^{2-}$, on $\mathrm{Li}(100)$. (d) $\mathrm{CO}_{3}^{2-}$ and $\mathrm{C}_{2} \mathrm{H}_{4}$ products on Li metal. The color key is the same as in Fig. 2. 

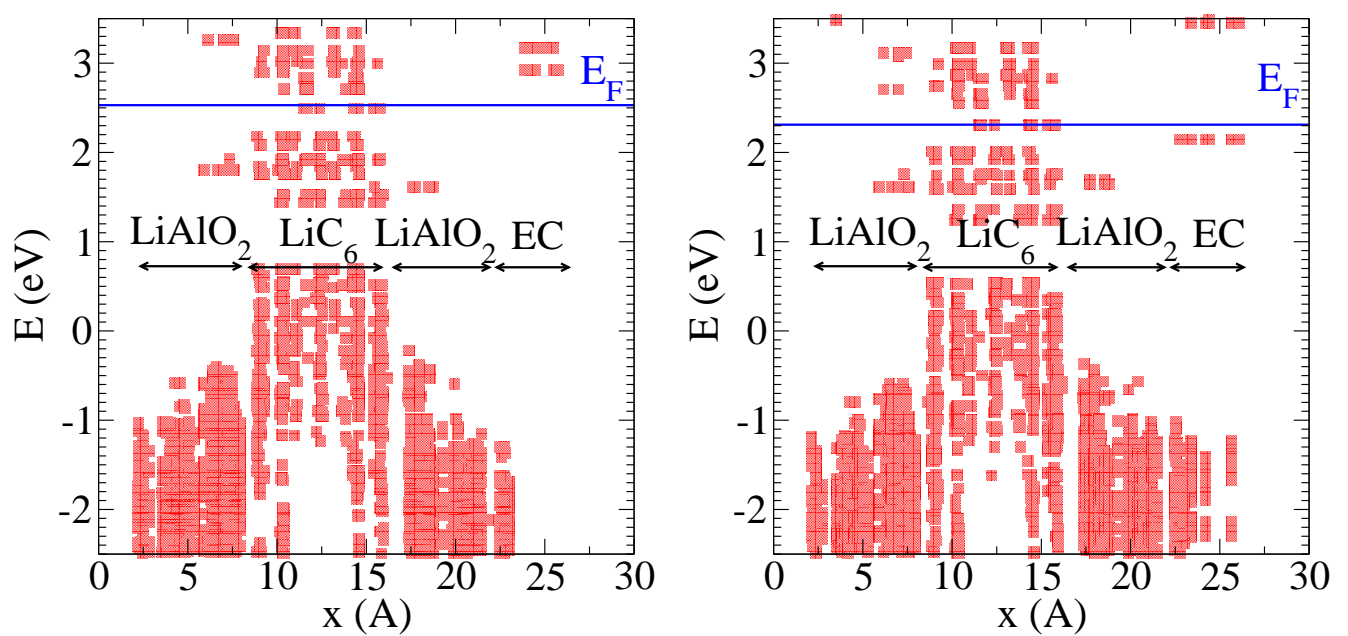

(c)
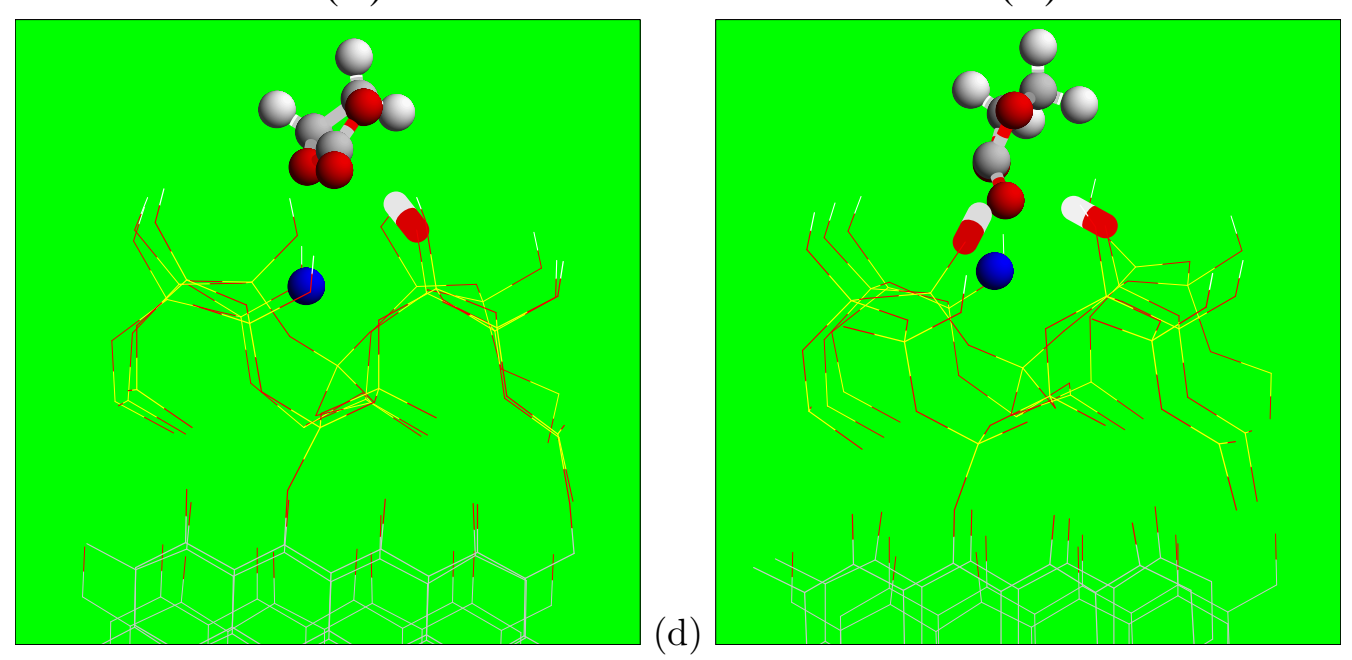

FIG. 4: (a)\&(b): Local electronic densities-of-state decomposed along the $x$-axis (perpendicular to interface) for EC adsorbed on thin $\mathrm{LiAlO}_{2}$-coated $\mathrm{LiC}_{6}$. Panels (a) and (b) correspond to the configurations depicted in panels (c) (flat EC geometry) \&(d) (bent geometry), respectively. The red patches depict integrated up- and down-spin densities exceeding $0.01|e|$ for each planewave wavefunction collapsed on an atom centered at $x$. Panel (b) shows that the bent geometry drastically changes the HOMO and LUMO levels, with an excess electron now residing on EC below the Fermi level $\left(E_{\mathrm{F}}\right)$. The conduction band of the $\mathrm{LiAlO}_{2}$ region is located above $4 \mathrm{eV}$. In panels (c) \& (d), the EC molecule and Li coordinated to the EC are depicted as spheres while surface hydroxyl groups donating hydrogen bonds to the EC are stick figures. Other Li are omitted and all other oxide-coating and graphite atoms appear as wireframes. EC configurations on the thick $\mathrm{LiAlO}_{2}$ coating are qualitatively similar (not shown). The color scheme is as in Fig. 2. 

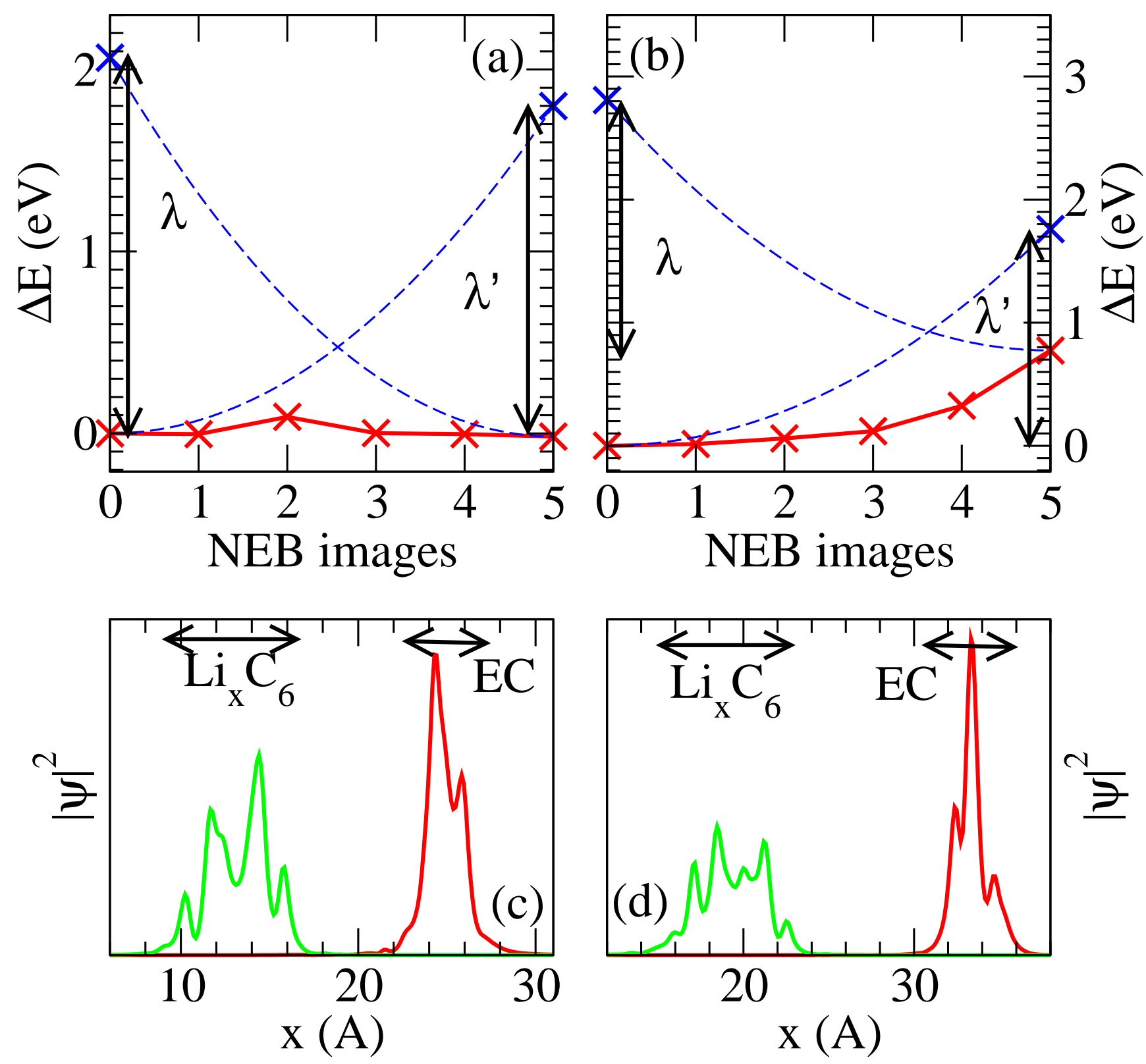

FIG. 5: (a)\&(b) Adiabatic (red crosses) and non-adiabatic (blue crosses) energy profiles along the reaction coordinate between the flat (Fig. 4k, image "0") and bent (Fig. 4d, image "5") EC geometries when applying a $0.4 \mathrm{~V} / \AA$ applied electric field to the $7 \AA$ and $10 \AA$-thick $\operatorname{LiAl}_{2} \mathrm{O}$ layers. Adiabatic energies are computed along the NEB-generated chain with unconstrained DFT. Non-adiabatic reorganization energies $(\lambda)$ derive from cDFT. The dashed curves are parabolic fits. (c)\&(d) Highest occupied orbital of the system (green), and the cDFT-computed $e^{-}$-accepting EC orbital (red) adsorbed on the $7 \AA$ and $10 \AA$-thick $\mathrm{LiAl}_{2} \mathrm{O}$ layers, integrated over the lateral dimensions. 

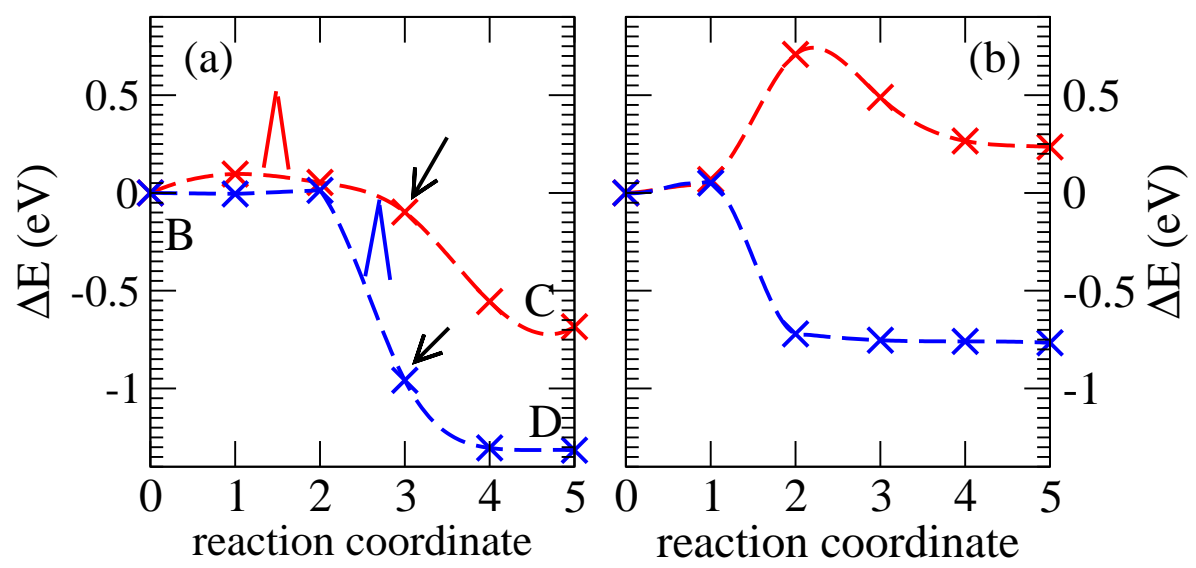

(c)
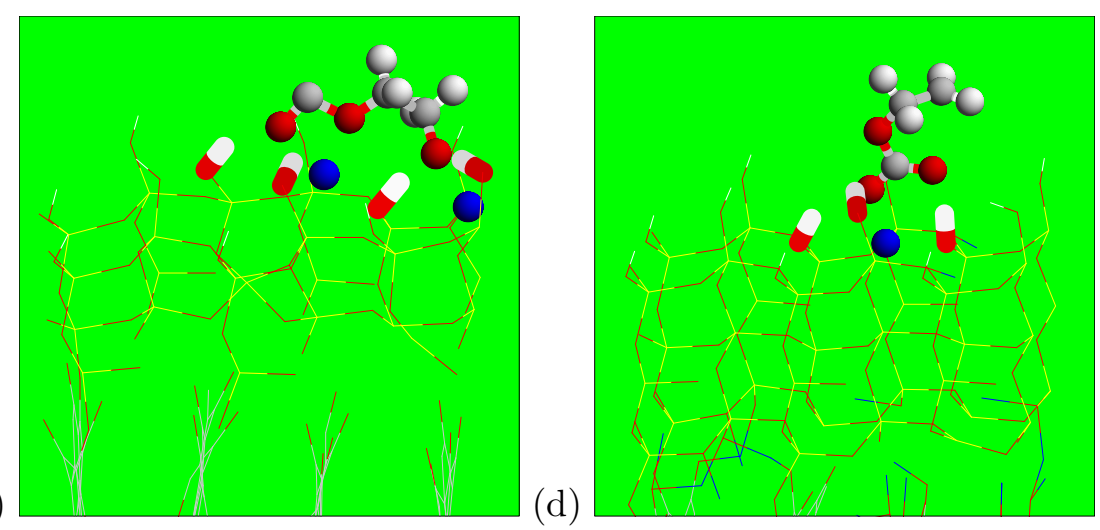

FIG. 6: (a) Adiabatic DFT/PBE energy profiles associated with EC decomposition on the thin $\mathrm{LiAlO}_{2}$ coating at $\mathrm{T}=0 \mathrm{~K}$. Red and blue refer to adsorbed $\mathrm{OCOC}_{2} \mathrm{H}_{4} \mathrm{O}^{2-}$ and $\mathrm{C}_{2} \mathrm{H}_{4} \mathrm{OCO}_{2}^{2-}$ intermediates, which are precursors to $\mathrm{CO}$ and $\mathrm{CO}_{3}^{2-}$ products, respectively. The electric field strength is $0.4 \mathrm{~V} / \AA$. The dashed lines are guides to the eye. Red/blue triangles depict the barrier associated with the first transfer of an electron on to the EC molecule, which is detected between images. They indicate that the DFT/PBE treatment erroneously neglects the electron tunneling barrier. Black arrows denote first detection of $\mathrm{C}-\mathrm{O}$ bond breaking in an image. Point $\mathrm{B}$ correspond to the intact EC in Fig. 4t. (b) Same as (a) but for a single $10 \AA$ thick layer in the absence of $\operatorname{Li}_{x} \mathrm{C}_{6}$ or electric field. (c)-(d) $\mathrm{OCOC}_{2} \mathrm{H}_{4} \mathrm{O}^{2-}$, and $\mathrm{C}_{2} \mathrm{H}_{4} \mathrm{OCO}_{2}^{2-}$ on thin $\mathrm{LiAlO}_{2}$ surfaces, corresponding to points C \& D in panel (a). The atom representation is as in Fig. 4 . 

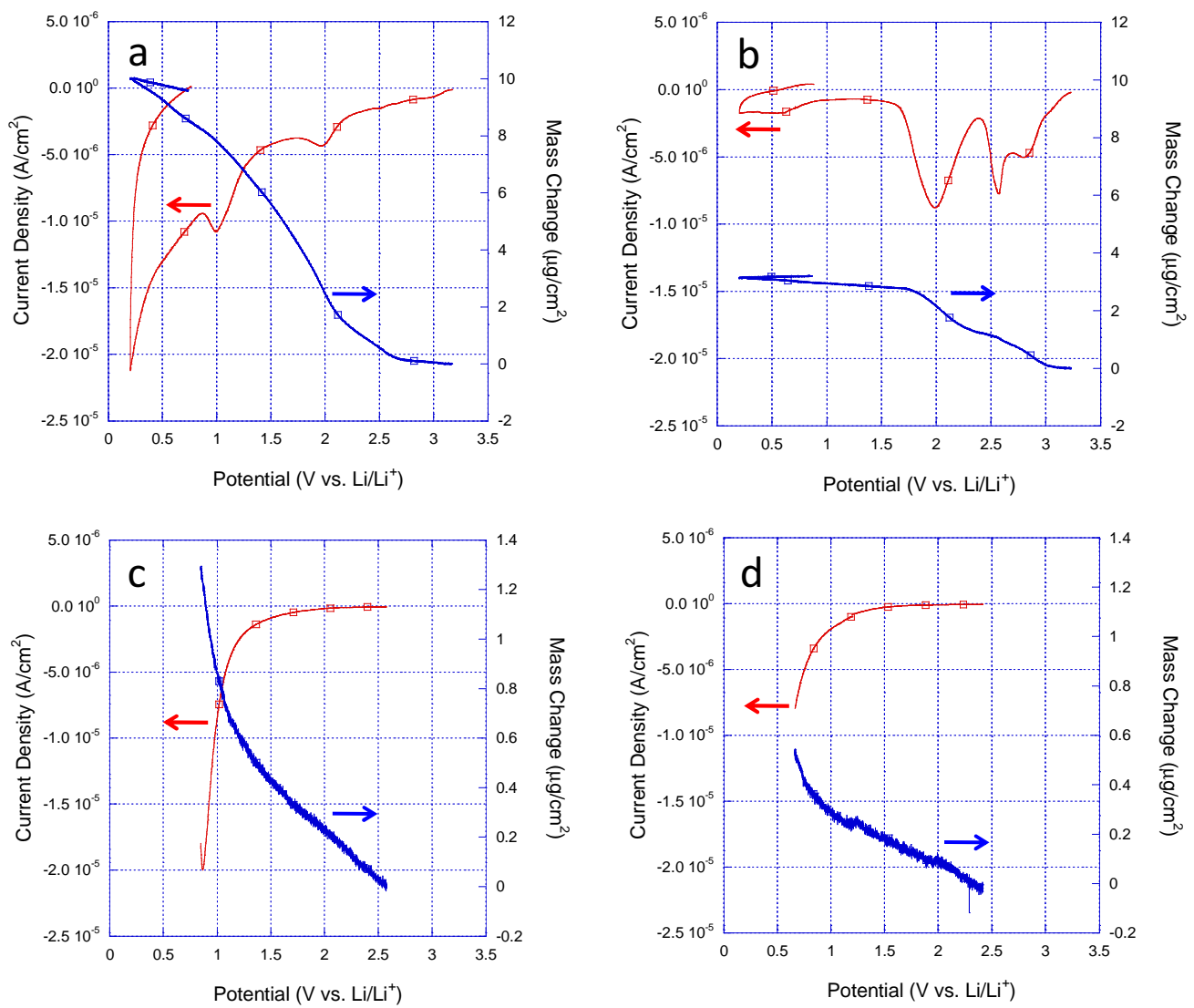

FIG. 7: Current and mass change response with $1 \mathrm{mVs}^{-1}$ cathodic polarization of an electrode in 1 M LiPF6, 1:1 vol. EC:DEC. (a) $50 \mathrm{~nm} \mathrm{C} \mathrm{film,} \mathrm{(b)} \mathrm{Cu} \mathrm{substrate} \mathrm{without} \mathrm{a} \mathrm{C} \mathrm{film,} \mathrm{(c)} 0.55 \mathrm{~nm}$ thick ALD $\mathrm{Al}_{2} \mathrm{O}_{3}$ on a $50 \mathrm{~nm} \mathrm{C}$ film, and (d) $1.1 \mathrm{~nm}$ thick $\mathrm{ALD} \mathrm{Al}_{2} \mathrm{O}_{3}$ on a $50 \mathrm{~nm} \mathrm{C}$ film. Current and mass are normalized to the geometric area. 